\title{
REVIEWS
}

\section{Dynamic Photoresponsive Functions in Organized Layer Systems Comprised of Azobenzene-containing Polymers}

\author{
Takahiro SEKI ${ }^{\dagger}$ \\ Department of Molecular Design and Engineering, Graduate School of Engineering, \\ Nagoya University, Chikusa, Nagoya 464-8603, Japan
}

(Received March 15, 2004; Accepted April 2, 2004; Published June 15, 2004)

\begin{abstract}
Azobenzene (Az)-containing polymers provide fascinating photoresponsive actuation systems. When they are organized at interfaces, the motion of Az unit occurring at a molecular (sub-nano) scale can be transferred and amplified to larger scales of materials. This review article focuses to selected research topics on organized layer systems of Az-containing polymers proceeding in our laboratory. They involve i) photomechanical response of monolayers at nanometer levels in thickness and centimeters in area, ii) monolayer-mediated photoorientation of polymer (polysilane) and mesoporous silica at nano- to sub-micrometer levels in thickness, and iii) instant mass migration triggered by patterned photoirradiation occurring at distances over micrometer levels. In every system, strong cooperative interplays between the Az unit and organized molecular or macromolecular assemblies play the essential roles. Promotions of these research areas are anticipated to provide wide and new opportunities for material processing and creation of new classes of photofuncitional soft materials. [DOI 10.1295/polymj.36.435]

KEY WORDS Azobenzene-containing Polymers / Organized Molecular Films / Interfaces / Photomechanical Effects / Photo-orientation / Polysilanes / Mesoporous Silica / Photo-triggered Mass Migration /
\end{abstract}

Actuations in biological systems have highly sophisticated hierarchical structures and work in ideal efficiency. In contrast, artificial actuation systems of soft materials are still at very primitive stages in terms of architectures of the constituents and effeiciency. Therefore, the final goal for researchers engaged in such field would be to produce artificial macromolecular systems closely mimicking biological muscle or motor systems. Thereby, actions and interplays at interfaces between macromolecular (protein) assemblies play the critical role. In this motivation, it is of no doubt that studies focusing on the design of mobile and actuating interfaces will be of particular significance.

Among various types of stimuli that may be utilized to trigger actions, light-driven motions and material controls are of fascinating subject. Light can approach to materials in non-contacting manners with high addressing accurately. In addition, various types of modulations are available in terms of intensity, energy (wavelength), polarization, coherency etc. In principle, molecular assemblies can receive all of such various types of modulating information. Also, when one adopts a photochromic molecule, reversible or repeatable functionalities are available.

Research on azobenzene (Az)-containing polymeric films has exploded during the past few decades, and it is continuing to be a very active area of materials chemistry. ${ }^{1-3}$ Azobenzene is perhaps the most frequently used photochoromic unit to be incorporated in molecular assemblies and organized polymeric materials. It has several advantageous features for fabrication of photoresponsive systems. i) Tedious synthesis is generally not required, leading to facile preparation of planned derivatives. ii) The sensitivity of trans $(E$-form $) \rightleftharpoons c i s$ ( $Z$-form) photoisomerization is moderately high for both directions for derivatives having alkyl or alkoxyl substituents. Besides, less expensive light sources such as Hg lamp can be used. iii) Photofatigue is negligible in ordinary experimental conditions laboratory. iv) The molecular shape is rodlike and symmetric, which favors incorporation into various molecular assemblies especially into liquid crystalline systems. ${ }^{4,5}$ In the light of above facts, photo "chromic" function is less important for Az unit, but photoresponsive functions to trigger motions are of particular significance. The photophysics and photochemistry of $\mathrm{Az}$ is summarized in literatures. ${ }^{6,7}$ Among many types of photofunctions of Az, this article only describes aspects of dynamic motions of molecules and optic and electric effects such as nonlinear optical response or photorefractive effects without alternation of molecular configuration are not dealt with.

${ }^{\dagger}$ To whom correspondence should be addressed (Fax: +81-52-789-4669, E-mail: tseki@apchem.nagoya-u.ac.jp). 
In the molecular design, connection of $\mathrm{Az}$ unit to a polymer chain is an essential strategy to realize effective photoresponsive systems. Needless to say, great improvement of film forming ability (processability) is one of the most important requirements, but for this chromophore use of polymers has even greater significance. Low-molecular-mass compounds tend to form crystals due to their high symmetry, which strictly inhibits reversible $c i s \rightleftharpoons$ trans photoisomerization. Introduction of amorphous nature in the materials favors efficient photoresponses. Importance of non-crystalline state for efficient photoisomerization of $\mathrm{Az}$ unit is well recognized for Az-containing LangmuirBlodgett (LB) films. ${ }^{8-10}$

In organized systems, the motion of $\mathrm{Az}$ unit taking place at a molecular (sub-nano) scale, can be transferred and amplified to larger scale levels of materials ranging from nanometer levels to over centimeter levels. This article reviews selected research activities on organized layer systems of Az-containing polymers undergoing in our laboratory including the backgrounds. ${ }^{11,12}$ They involve i) photomechanical response of monolayers (nano-levels in thickness and centimeters in area), ii) monolayer-mediated photoorientation of polymer (polysilane) and mesoporous silica (nano- to sub-micrometer levels in thickness), and iii) finally instant mass migration triggered by patterned photoirradiation (over micrometer levels). In every system, the interplay between Az unit and organized molecular or macromolecular assemblies is of essential and exploration in these areas are anticipated to provide wide and new opportunities for creation of new classes of photofuncitional soft materials.

\section{PHOTOMECHANICAL RESPONSE IN MONOLAYER SYSTEMS}

\section{Macro-size Photomechanical Effects}

In a bulk state, molecular motions in response to light cannot be directly reflected in the macro-size behavior because of structural complexities and hierarchies existing in the materials. ${ }^{13}$ In the monolayer state, on the other hand, changes in the molecular shape and orientation can be simply and directly related to the film properties such as film area and surface pressure. In this regard, lowering the dimensionality from three dimensions (3D) to two dimensions (2D) is particularly meaningful. Chemical structures of Az-containing polymers studied for photomechanical response in the monolayer state are displayed in Figure 1.

Photoresponsive effects in Az-containing polymers were first reported by Blair et al. ${ }^{14,15}$ using polyamids having an $\mathrm{Az}$ unit introduced in the main chain ( $\mathbf{1}$ in Figure 1). Langmuir Film balance measurements show an area reduction on changing from dark to UV light exposed conditions resulting from the trans to $c$ is isomerization. Anormalous expansions are observed for the $4,4^{\prime}$-substituted polymer. The area change upon UV light irradiation is quite complicated. The monolayer film initially contracts which is subsequently followed by area expansions at constant pressure of less than $10 \mathrm{mN} \mathrm{m}^{-1}$.

A photoresponseive polypeptide monolayer on water was first reported by Malcolm and Pieroni. ${ }^{16}$ Photoresponsive polypeptide systems may provide useful implications for understanding the mechanisms operating in biological photoreseptors. Poly(L-lysine) containing ca. $40 \mathrm{~mol} \% \mathrm{Az}$ units in the side chains ( 2 in Figure 1) forms a stable monolayer on water. When the polypeptide monolayer is kept at constant area, irradiation at $365 \mathrm{~nm}$ produced a decrease of the surface pressure, which reversibly reverts to the original value upon irradiation at $450 \mathrm{~nm}$. At a constant pressure, irradiation with $365 \mathrm{~nm}$ and $450 \mathrm{~nm}$, alternately, produces reversible changes of the surface area of the monolayer. Infrared spectrum indicates that the $\alpha$-helix structure is retained regardless of light irradiation. ${ }^{17}$ This suggests that in the monolayer state the polymer does not undergo a confomational change on irradiation. The photomechanical effects seem to be simply due to the trans-cis isomerization of the Az groups. Ahluwalia et al. ${ }^{18}$ conducted surface potential measurements for this monolayer. The data can be quantitatively analyzed by the inverted cone model. ${ }^{19}$ The angle of dipole inclination for the trans and $c i s$ isomers of the Az side residue is estimated as a function of area per residue.

Photoresponses of related Az-containing polypeptides were investigated by Menzel. ${ }^{20} \mathrm{He}$ prepared poly(L-glutamate)s containing Az side chains which are in a category of 'hairy rod' polymers (3 in Figure 1). ${ }^{21,22}$ Interestingly, these monolayers show an expansion upon UV light irradiation, which is the opposite direction as the above mentioned monolayer for the poly(L-lysine) type. Thus, the photomechanical effect is very sensitive to the architecture of the polymers. From a comparison of the isomerization rate obtained from the spectroscopic measurements and the rate of the photomechanical response, it can be deduced that the photomechanical effect is not directly caused by the photoisomerization. Instead, this twostep mechanism for restructuring the monolayers upon irradiation has to be assumed. When the Az moiety is transformed from trans to cis, this chromophore moves to the water surface and contribute to the surface area per monomeric unit. This interpretation is in essential consistency with that observed for $\mathrm{Az}$ pendant poly(vinyl alcohol)s studied by Seki et al. as will be described below. 


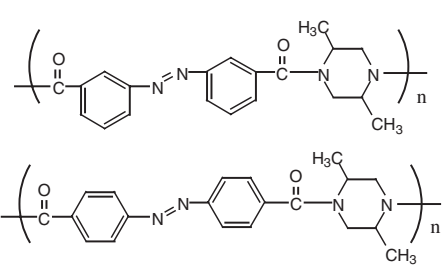

1

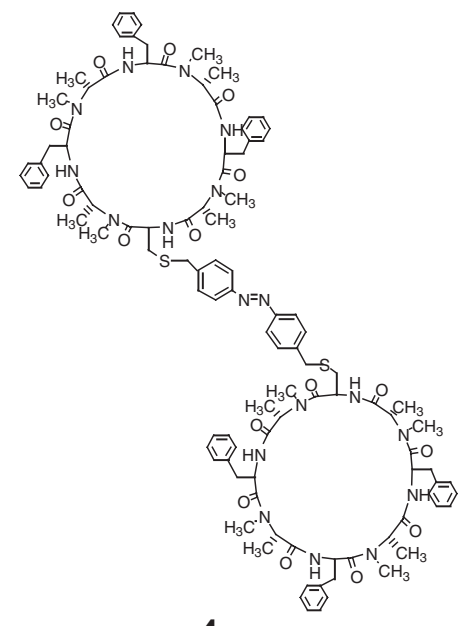

4
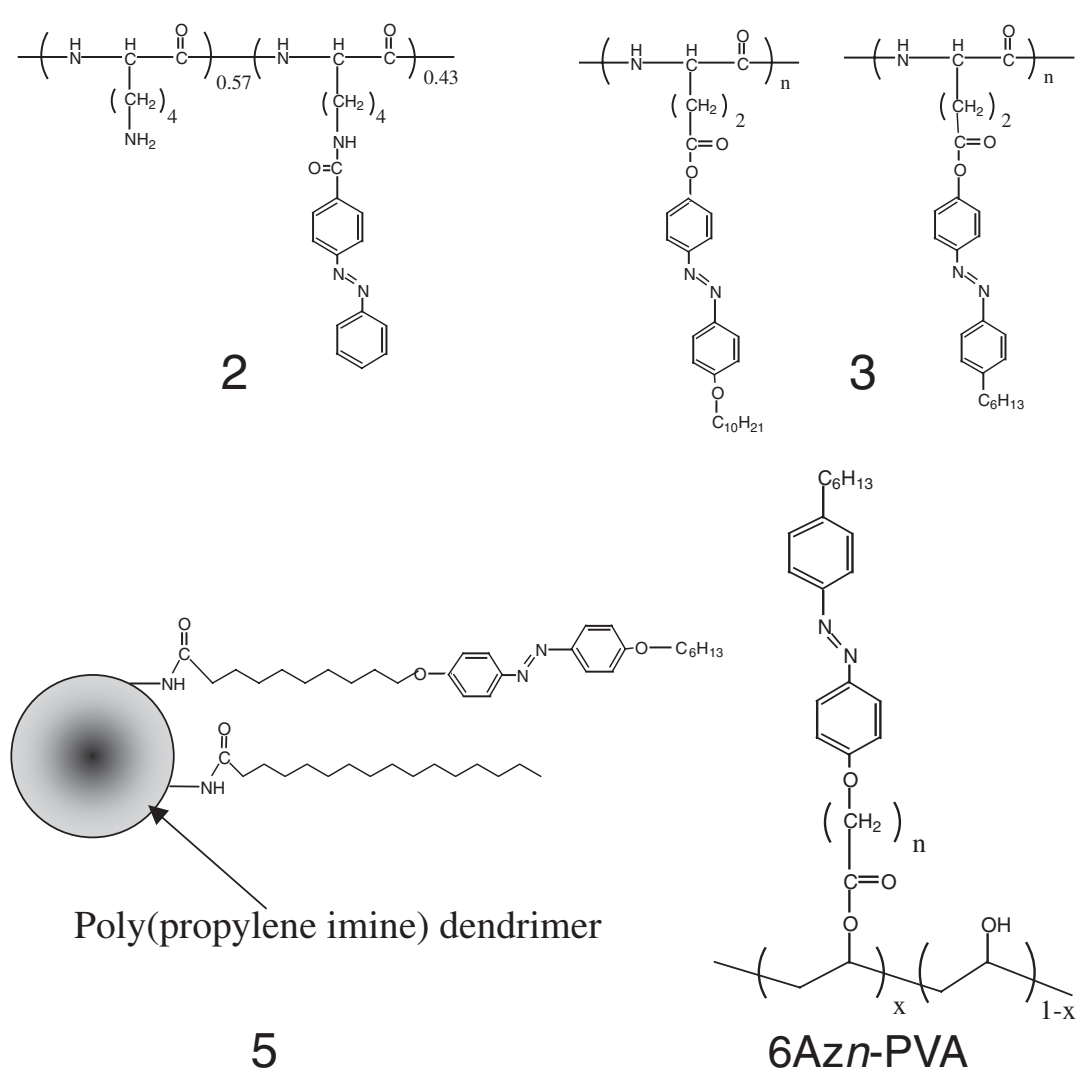

Figure 1. Typical Az-containing polymers investigated for photomechanical effects in monolayers.

Ghadiri's group ${ }^{23}$ reported on the photoresponsive behavior of an Az derivative possessing two cyclic octapeptides (4 in Figure 1). ${ }^{24}$ The IR spectroscopy shows that the peptide cylinders are oriented predominantly perpendicular to the surface normal. In both trans- and cis-forms, this cyclic peptide derivative provided a stable monolayer with some difference in the plateau regions depending on the isomeric form. The surface in the plateau region, a second peptide layer is formed as revealed by scanning force microscopy.

Weener and Meijer ${ }^{25}$ examined spreading and photoresponsive behavior of poly(propylene imine) based dendrimers possessing Az unit at the terminals. Reversible photoisomerization occurs for a fifth generation polymer terminated with both $\mathrm{Az}$ and $\mathrm{C} 16$ long chain (50:50). Photoinduced area change is observed for this polymer. On the other hand, a dendrimer in which $\mathrm{Az}$ unit is fully introduced is not suited for monolayer experiment.

Azobenzene-containing dendrons with a crown ether head have also been spread at the air-water interface by Sidorenko et al. ${ }^{26}$ The photoinduced area increase upon UV light illumination is examined as a function of dendron generation. The generation of one and two leads to considerable area increases (10-20\%), however, higher and lower generations demonstrated minor changes (5-10\%). This can be as- cribed to different lateral compliant properties of compound having different numbers of alkyl chains with radial orientation.

Seki et al. ${ }^{27-29}$ prepared poly(vinyl alcohol)s (PVA) having an Az-containing side chain (6Azn-PVA in Figure 1) and observed a photoinduced area changes on a water surface. The magnitude of the film deformation is the function of spacer length connecting the Az moiety and the PVA backbone. Irradiation with $365 \mathrm{~nm}$ light brings about a rapid expansion of the monolayer. The largest effect is observed for 6Az10-PVA. The film exhibits a ca. 3-fold expansion from the original area. When $436 \mathrm{~nm}$ light is irradiated, the film shows a rapid contractile motion to the initial area. The repetition was achieved with full reproducibility. The Az in the cis-form is much polar than the trans-form, which brings about a contact with the water surface. This leads to the expansion of the monolayer. The reversible process takes place upon visible light irradiation (Figure 2). This model is reasonably understood by dependence of the spacer length. In-situ $\mathrm{X}$-Ray reflectivity analysis on the water surface was performed by Kago et al. ${ }^{30}$ for 6Az10-PVA. Kiessig fringes are observed for specular measurement for 6Az10-PVA monolayers on a water surface. By analyzing the XR data, it is indicated that the thickness of the monolayer became larger for the trans form than the cis form. The thickness change by $0.2-0.3 \mathrm{~nm}$ 


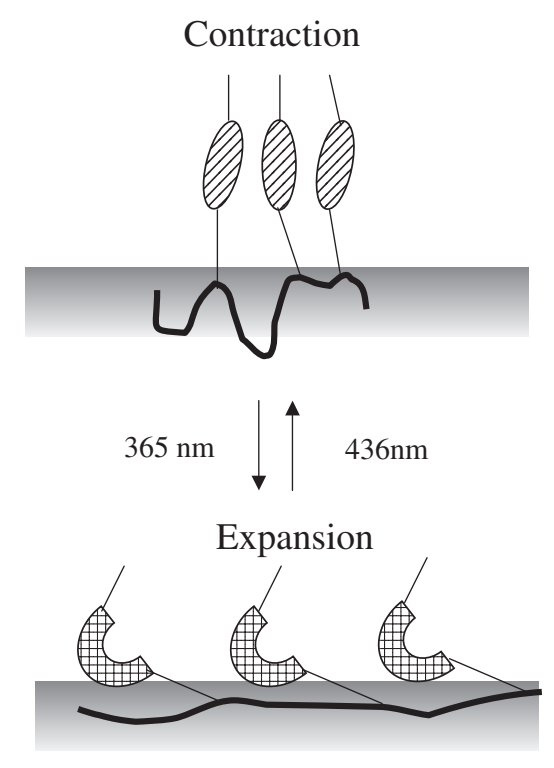

Figure 2. Schematic illustration of photoinduced expansion and contraction of 6Azn-PVA monolayer on water.

due to the trans-cis configurational change in the hydrophobic side chain is directly proven on the water surface. $^{30}$

Wüstneck et al. ${ }^{31,32}$ adopted a unique approach for estimation of the photoinduced change of an amphiphilic Az containing polymer in surface pressure using a pendant water drop. The surface tension of the drop was determined by axisymmetric drop shape analysis. ${ }^{33}$ This method readily allows for determination of surface dilatational parameters such as surface dilatational elasticity, relaxation time, and surface dilatation viscosity by a stress relaxation experiments. The differences in the rheological properties in the trans- and cis-Az containing monolayers are revealed.

\section{Visualization of Polymer Motions by Microscopic Ob-} servations

Macro-size mechanical responses at $10^{-1}-10^{-3} \mathrm{~m}$ levels can be evaluated by film balance measurements. On the other hand, motions and structural changes at molecular $\left(10^{-9}-10^{-10} \mathrm{~m}\right)$ levels can be evaluated by absorption spectroscopy, X-Ray reflectivity and surface potential measurements. Thus, there exists a tremendous scale gap between the observation tools where many hierarchical structures are involved. To bridge the two scale regimes, microscopic visualization methods are of particular importance. The dynamism of the photoresponsive monolayers has been actually revealed by visualization tools. Optical microscopic tools such as fluorescence microscopy and Brewster angle (ellipsometric) microscopy (BAM) cover scale ranges around $10^{-3}-10^{-6} \mathrm{~m}$ levels. Atomic force microscopy (AFM) visualizes smaller ranges from micrometers to molecular levels. In-situ observa- tions on the water surface which reflects the "living" state are feasible by the optical microscopies, and in contrast, the transfer process onto a solid substrate is required to perform AFM observations. Both methods are very important and complementary visualization tools to cover the whole length scales.

BAM utilizes the propagation and reflecting properties of light. When $p$-polarized light is incident on the water surface at the Brewster angle, no light is reflected. In a case that a floating monolayer exists, the Brewster angle conditions are no longer valid due to a change in the reflective index at the surface and a small portion of light is reflected. ${ }^{34,35}$ This method is well suited for observation of photochromic monolayer systems because no additional photoexcitable molecule is required. In addition, when a $\mathrm{He}-\mathrm{Ne}$ laser beam is used, the probing light $(633 \mathrm{~nm})$ scarcely influences the photoprocess of both isomerization directions of Az.

Seki et al. ${ }^{36,37}$ have utilized BAM to clarify the nature of photomecanical response in monolayer using 6Az10-PVA, focusing on the following points: 1) changes in the rheological properties due to the photoisomerization, 2) in-situ observation of photoinduced area changes at microscopic scales, 3) evaluation of photoresponsive behavior at zero pressure (inherent behavior), and 4) precise evaluation of time correlation between the phototrigger and film response. For these purposes, a small mobile trough that can chase an isolated piece of photoresponsive monolayer within the microscope field has been constructed. With this setup, the photoinduced area changes with time are directly followed from the BAM image (Figure 3). ${ }^{37}$ An important observation is that an induction time is clearly observed before the monolayer starts expanding despite the fact that the trans-to-cis photoisomerization proceeds just after the switch-on point of illumination. The expansion occurs after more than $40 \%$ of the $\mathrm{Az}$ side chains are isomerized to cisform. This fact can be the consequence of an antagonistic action between the expanding action of cis-isomerized side chains and contracting (aggregating) effect of the trans side chains. Thus the mechanical process involves strong molecular cooperativity. The nonlinearity becomes much more pronounced when a surface pressure is applied. At $2 \mathrm{mN} \mathrm{m}^{-1}$ the expansion starts after the monolayer system almost reaches to its photostationary state. The nonlinear property in the expansion process depends on the spacer length. The longer alkylene spacer gives rise to stronger nonlinearity due to the stronger aggregation action in the trans form. ${ }^{38}$

Photoinduced morphological changes at molecular levels may be visualized by atomic force microscopy (AFM). Actually, large distance migrations of a poly- 

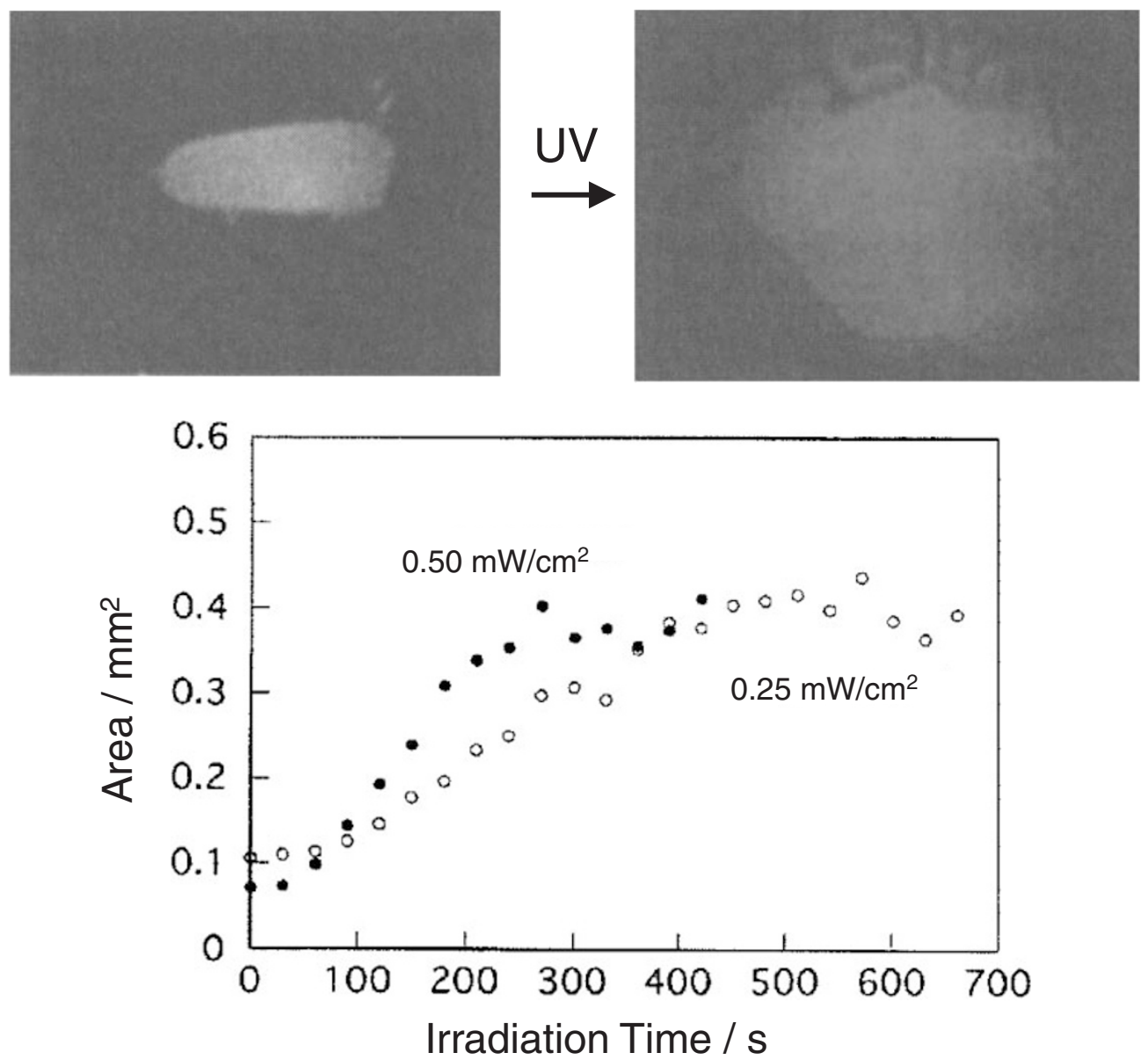

Figure 3. In situ observation of photoinduced expansion of 6Az10-PVA by BAM (upper) and time course of area change $v s$. irradiation time (lower). Note that the expansion indicates nonlinear cooperative behavior.

mer monolayer in relation to mechanical response are indicated with 6Az10-PVA monolayer by AFM. ${ }^{39,40}$ It is found that the morphology of the 6Az10-PVA monolayer on a hydrophilic mica surface changes by illumination in a highly humidified atmosphere. Clear humidity dependence of the morphological change is observed for a film transferred at a lateral density of $1.2 \mathrm{~nm}^{2}$ per $\mathrm{Az}$ unit. In a dry state (relative humidity (RH) below 25\%), the flat morphology is maintained. At higher humidity, the monolayer undergoes larger lateral movement to form network structure at micrometer levels. Thus the motions of the monolayer are assisted by water molecules existing on the hydrophilic surface. ${ }^{41,42}$ The UV light irradiation induces 2D expansion of the layer, which is then reverted to an essentially the identical contracted morphology after thermal reaction spending for a few days. When the layer is more densely packed $\left(0.4 \mathrm{~nm}^{2}\right.$ per Az unit), the layer shows a sponge-shaped structure with a number of defects. Upon UV light illumination, most of the defects disappear and instead huge shallow panlike protrusions of $200-300 \mathrm{~nm}$ in diameter and $c a$. $10 \mathrm{~nm}$ in height appear. This 3D change is also nearly a reversible process. The $3 \mathrm{D}$ protrusions probably consisted of bubbles instead of multi-layering from the following facts.

Matsumoto et al. ${ }^{43,44}$ have prepared LB films consisting of an ion complex formed between an anionic $\mathrm{Az}$ derivative and a cationic polymer. A very flat surface in the ion-complex LB monolayer on mica is observed before illumination. After UV light illumination the morphology drastically changes to form a number of hills having diameter of $c a .100 \mathrm{~nm}$ with the height of $5 \mathrm{~nm}$. The highly undulated structure reverted to the original flat surface after irradiation with visible light. The humidity conditions are not mentioned for this work, but their observations are in essential coincidence with Seki's observed at a highly dense packing state. ${ }^{40}$ Thus, an interpretation of the photoisomerization behavior of $\mathrm{Az}$ based on the free volume is not hold in such soft film systems.

As an extremely diluted state, the 6Az10-PVA monolayer was isolated to a single chain level. ${ }^{45}$ To avoid complexities as mentioned above, observation of intrinsic photoresponse of the monolayer isolated ideally to a single chain level is highly desired. Segregation to the level of the single polymer involvement may be achieved by high dilution of solutions ${ }^{46}$ or in 


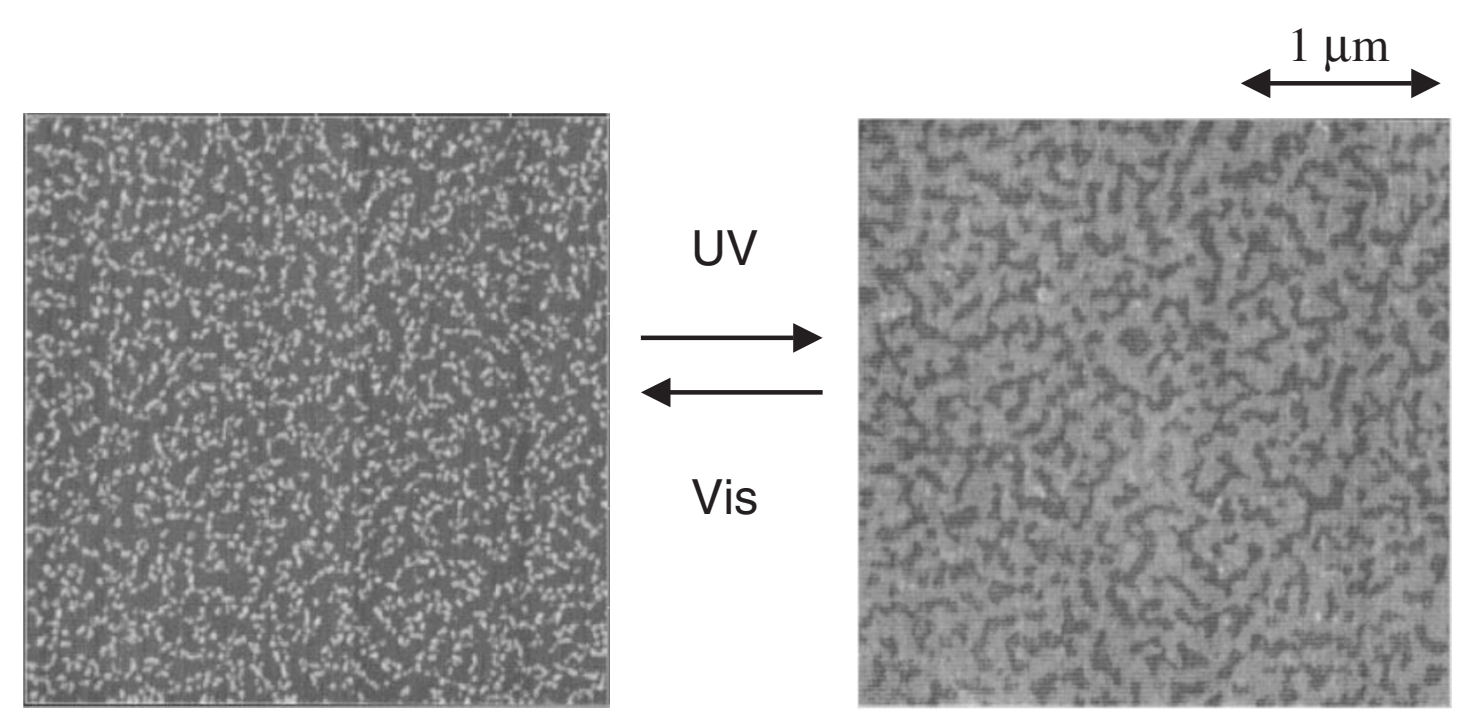

Figure 4. Topological AFM images showing reversible expansion and contraction of 6Az10-PVA monolayer on mica. The monolayer is two dimensionally diluted via skeletonization with stuffing amphiphile (see text).

monolayers. ${ }^{47}$ For 6Az10-PVA monolayer, however, the lipophilic long side chain strongly promotes aggregation and prohibits isolation to a single chain. In such case, the process of so-called skeletonization of the LB monolayer is effectual. Skeletonization involves selective removal of one component from a mixed organization. ${ }^{48,49}$ 6Az10-PVA is mixed with a stuffing amphiphile (oleic acid or methyl oleate) that shows a high expansion at the air water interface. After transfer onto a mica surface at very low pressures, the stuffing material is then removed by washing or evaporation in reduced pressures at elevated temperatures. Dot shaped films having $c a .20-30 \mathrm{~nm}$ radius are obtained consequently. Volume estimations and some considerations lead to an aspect that the dot films are consisting of single polymer chain or at least separated at a comparable level. The in-situ AFM measurement indicates the actual expansion (after UV light irradiation) and contraction (visible light) behavior of the isolated polymer monolayer accompanied by a thickness reduction from $1.2 \mathrm{~nm}$ to $0.5 \mathrm{~nm}$, which reasonably agrees with the model shown in Figure 2. These motions are fully reversible. A more crowded ensemble also indicates fully reversible morphological changes between a completely isolated film in the trans state and continuous film formation in the cis state (Figure 4). This fact implies that the polymer chains restricted in the 2D state cannot be entangled with each other. This reasonably accounts for the rapid separation and reproducibility of the dot structure.

\section{Photocontrol of Two Dimensional Phase Separation \\ Hybrid monolayers consisting of 6Az10-PVA and a polar liquid crystal (LC) molecule, 4'-pentyl-4-cya-}

nobiphenyl (5CB) provide a good interface model of the command surface for photoswitching of LC alignment. ${ }^{4}$ A strong molecular cooperativity and essential factors of orientation switching behavior resulted by the photoisomerization of $\mathrm{Az}$ have been revealed by this approach. ${ }^{50-52}$ The surface pressure-area $(\pi-\mathrm{A})$ isotherms obtained at various mixing ratios $(r=$ $[5 \mathrm{CB}] /[\mathrm{Az}$ units] $)$ indicate that one $\mathrm{Az}$ side chain can accommodate two 5CB molecules. Above this critical ratio, thinner parts consisting of pure 5CB monolayer start to grow to show a two-dimensional phase separation.

Topographical AFM images of mixed monolayers and its analysis reveal that the phase separated structure consists of thicker monolayered domains involving two 5CB molecules per Az unit and thinner ones comprising pure $5 \mathrm{CB}$ monolayer. ${ }^{53}$ The reason for the capacity to accommodate two 5CB molecules with one Az unit can be as follows. One 5CB molecule can be inserted into the spatial cavity formed between the $\mathrm{Az}$ unit and the backbone (spacer part) and the other can be positioned on the air side of the 6Az10 side chain (tail part). In situ X-Ray reflectometry shows that the 6Az10-PVA/5CB hybrid monolayer $(r=1)$ is composed of a dense lower layer and a less dense upper layer, indicating that $5 \mathrm{CB}$ molecules are inserted into the spatial cavity of the $\mathrm{C}_{10}$ spacer part. ${ }^{54}$

Figure 5 shows photoinduced morphological changes of the monolayer of 6Az10-PVA/5CB at $r=$ 4 deposited on a mica as revealed by AFM. The film is first irradiated with UV light and then with visible light under highly humid conditions. After UV light irradiation, virtually all domains disappear, resulting in smoother film with a surface roughness within $0.2 \mathrm{~nm}$ (b). Subsequent visible light irradiation regen- 
a

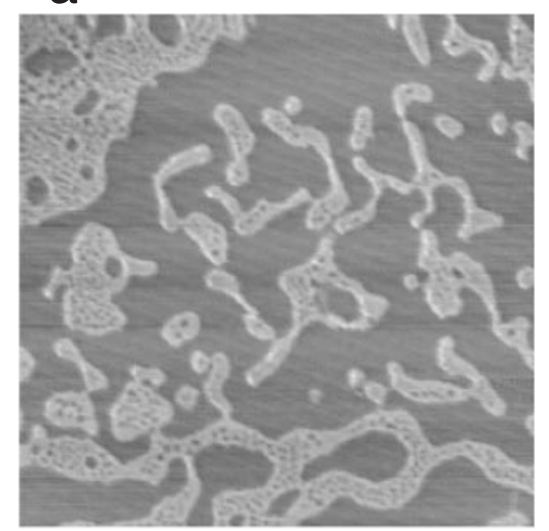

b

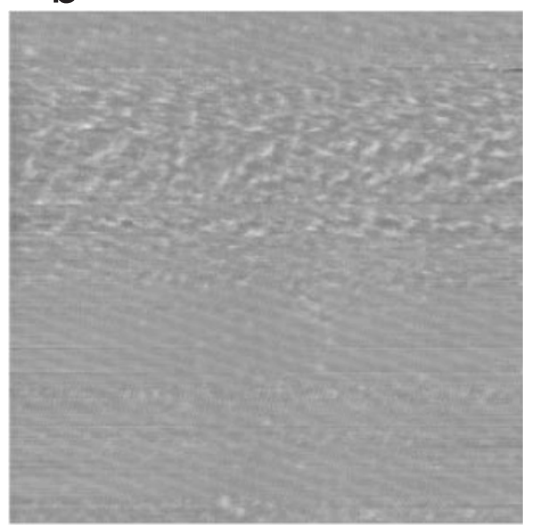

C

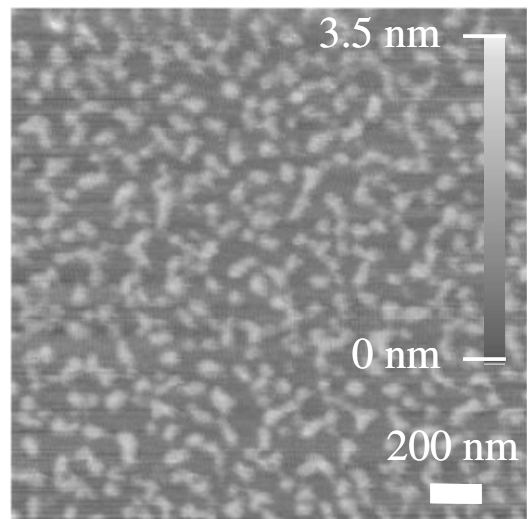

Figure 5. Photocontrol of phase separation behavior of 6Az10-PVA/5CB monolayer on mica $(r=4)$ : (a) as-transferred from water, (b) after UV light illumination, and (c) after visible light illumination.

erates the thicker domains of $50-100 \mathrm{~nm}$ in diameter and approximately $1.5 \mathrm{~nm}$ in height (c). Although the size of each projected domain becomes smaller, phase separation occurred again. The round domains are thicker than the thinner regions by $1.5 \mathrm{~nm}$, indicating good coincidence with those of the initial state before photoirradiation. It is to be noted that the change in morphology after the photocycle unequivocally shows that the polymer chain can migrate at micrometer levels. This may be related to photo-triggered large-distance mass migration as will be mentioned below.

On the mixing of a polymer with $5 \mathrm{CB}$, Nagano et al. have found that the hybridization of 5CB and a polysilane, poly(di- $n$-hexylsilan) (PDHS) on the water surface provides very interesting aspects in regard to surface physical chemistry of polymers. ${ }^{55,56}$ When a mixed solution of PDHS and 5CB is spread onto a water surface, an ideally extended monolayer of PDHS is spontaneously formed on 5CB monolayer. The monolayer of PDHS can be prepared on water despite the fact that the polymer is fully hydrophobic. Layer-bylayer transfer of the monolayer onto a substrate further leads to valuable information on the conformational behavior of the polymer. ${ }^{57}$ Although this knowledge gives new implications in polymer nano-processing, details are not described here because this topic is somewhat deviated from the framework of this article.

\section{Supramolecular Approach Using Urea Derivatives}

Supramoleuclar architecture formed through hydrogen bonding is now occupying a major research area in polymer chemistry. ${ }^{58,59}$ It seems of value to conduct such approach in the photoresponsive monolayer systems instead of employing polymers. Here, urea-containing $\mathrm{Az}$ derivatives are adopted (Figure 6). ${ }^{60-64}$ Urea is chosen as the hydrophilic head because the monolayer of alkylureas shows interesting characteris- tic properties attributed to the formation of so-called bifurcated (double) hydrogen bond among the urea units on water. ${ }^{65-68}$

When urea is connected with Az unit, further interesting behavior appears as follows. 1) The trans-to-cis photoisomerization of $\mathrm{Az}$ is completely hindered at the air-water interface despite the fact that the absorption maximum of the $\pi-\pi^{*}$ band almost coincides with that in solution. ${ }^{60}$ 2) A transferred monolayer on a hydrophilic substrate shows characteristic humidity-sensitive spectral change due to alternations of the 2D packing state in a reversible manner. ${ }^{61,69}$ This may provide an interesting molecular system for environmental sensors. 3) Obvious and multifarious carbon parity (even-odd) effects are observed with respect to carbon number of the spacer connecting the $\mathrm{Az}$ moiety and urea in both monolayers and multilayer systems. ${ }^{63,64}$

The crucial role of the bifurcated hydrogen bonding for the appearance of above features is clearly indicated by the chemical modification of urea moiety. ${ }^{62}$ Figures 7 displays the area changes of the monolayers of 6Az10-urea, 6Az10-UM, 6Az10-UM2, and $6 \mathrm{Az} 10 \mathrm{COOH}$ upon irradiation with UV and visible light at $20^{\circ} \mathrm{C}$ and $2 \mathrm{mN} \mathrm{m}^{-1}$. As seen from Figure 7, no photoreaction and thus no photoinduced area change are observed in the 6Az10-urea and 6Az10UM monolayers. In sharp contrast, the spectrum of 6Az10-UM2 monolayer exhibits reversible area changes under alternate irradiation. Also, the 6Az10-COOH monolayer behaves in the same manner. The discrepancy is attributed to the mode of hydrogen bond formation, namely, the above two monolayers can form the bifurcated hydrogen bonds, whereas the rest two form only single strand hydrogen bond (see the cartoons in Figure 7).

Odd-even effects in Langmuir monolayers found by Kobayashi et al. are particularly worth mentioning 


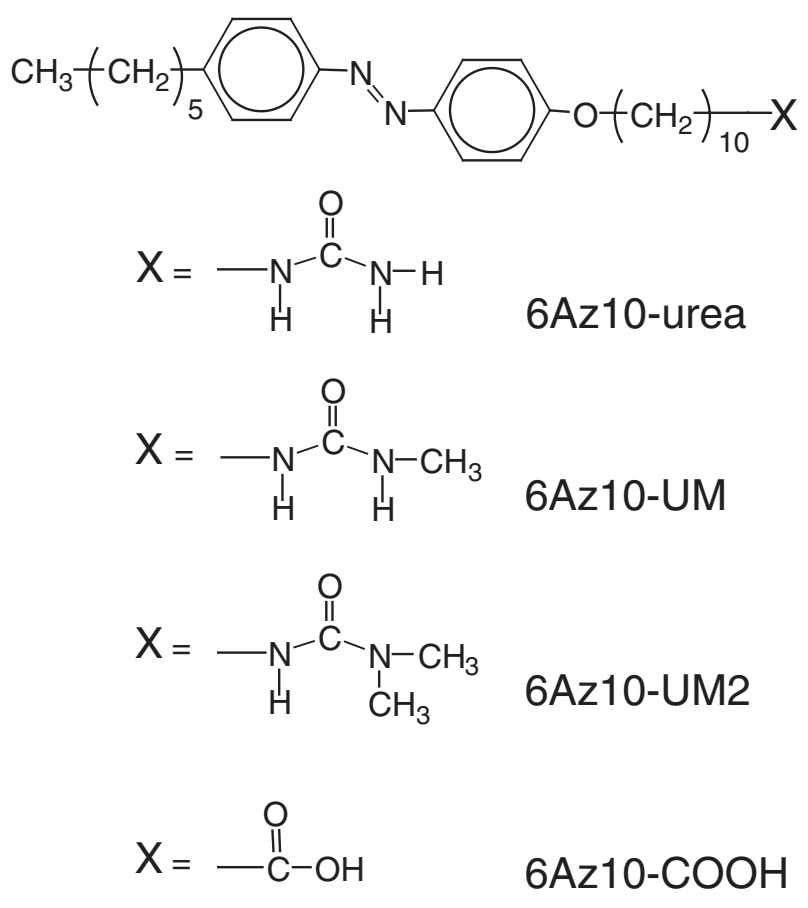

Figure 6. Az-containing urea derivatives and homologous carboxylic acid derivative.

(Figure 8). ${ }^{63,64}$ In the $\pi-\mathrm{A}$ isotherms of $6 \mathrm{Az} n$-urea $(n=3-7)$ in the trans form, the limiting area per molecule, which is estimated by extrapolating the steepest slope to zero pressure, indicate systematic alternations with $n$ (figure in the left). For the compounds of odd carbon numbered spacer $(n=3,5$ and 7), the limiting area ranges $0.29-0.30 \mathrm{~nm}^{2}$. On the other hand, the monolayers of 6Azn-urea having the even numbered spacer ( $n=4$ and 6 ) shows significant area expansions with higher compressibility. Since the cross section of vertically aligned $\mathrm{Az}$ is $0.25 \mathrm{~nm}^{2}$ as estimated from the X-Ray diffraction data of crystals, ${ }^{70}$ it can be thus interpreted that the molecules in these monolayers are tilted with respect to the surface normal. The degree of molecular tilt for the even numbered carbon spacer should be greater than that of the odd numbered ones. This is, to our knowledge, the first example to indicate carbon parity effects even in the monolayer state floating on a highly fluid water surface.

The right side of Figure 8 shows absorption spectra of the 6Azn-urea monolayers on a hydrophilic quartz plate. The monolayers of 6Azn-urea transferred on a hydrophilic substrate give spectra showing $\mathrm{H}$-aggregate formation of Az. Here, the spectra of the transferred monolayers indicate zigzag alternations of $\lambda_{\max }$ $\left(\pi-\pi^{*}\right.$ absorption in the long axis transition) with increase of the carbon spacer (closed squares in the inset of right figure). The alternations in the molecular orientation in these monolayers are also suggested from the spectra. The ratio of absorption intensity of two peaks, $A_{242} / A_{\text {long }}$, where $A_{242}$ and $A_{\text {long }}$ being absorbances at $242 \mathrm{~nm}$ and $\lambda_{\max }$, respectively, are plotted against the carbon number of spacer (open circles in the inset of Figure 8). This absorbance ratio can be a measure of molecular tilt. The smaller value of $A_{242} / A_{\text {long }}$ indicated the larger molecular tilt from the surface normal in the transmission mode measurements. The results obtained here indicate that the monolayers of even carbon numbers are tilted to more extents, which are consistent with the data of molecular occupying area in the spreading behavior. The alternations in the molecular tilt are also consistent with other data of X-Ray analysis and infrared spectroscopy obtained for multilayers. ${ }^{64}$

\section{SURFACE-MEDIATED CONTROL OF MATERIALS ON AZ MONOLAYER}

The surface-mediated photochemical alignment switching of nematic LCs on an Az-containing monolayer (command surface) was first reported by Ichimura et al. ${ }^{4,71}$ The application to LC systems is the earliest and widely examined example of the surface-mediated photocontrol, ${ }^{72}$ and, perhaps provides the most smart and practical systems due to the highly cooperative assembling nature of these anisotropic fluid matter. This section introduces our attempts to photo-align organized materials other than typical LCs, namely, polysilane and mesoporous silica.

\section{Conformational and Orientational Control of Poly- silane Films}

Light triggered alignment of polysilane has been initiated by Fukuda et al. ${ }^{73-75}$ A polysilane is employed due to its versatile optical property, namely the strongly light absorbing property attributed to the $\sigma$-electron conjugation. ${ }^{76}$ The absorption property is strongly dependent on the backbone conformation and directional, being parallel to the backbone extension. PDHS is particularly suited since the optical properties coupled with the conformational state is already well investigated. ${ }^{76}$

A spincast film of PDHS (ca. $60 \mathrm{~nm}$ thickness) is prepared on a 6Az10-PVA monolayer (Figure 9). The crystallization process after casting can be readily monitored from the spectral change. The crystallization rate is accelerated on the cis-Az monolayer, and retarded on the trans-Az monolayer. ${ }^{73}$ Most probably, the nucleation process at the interface between the PDHS film and the Az monolayer is altered by the photoisomerization, which then result in the crystallization control of the whole film. This fact provides an important issue that the photoersponsive molecular surface can control the physical state throughout the entire thickness of the polymer film. 


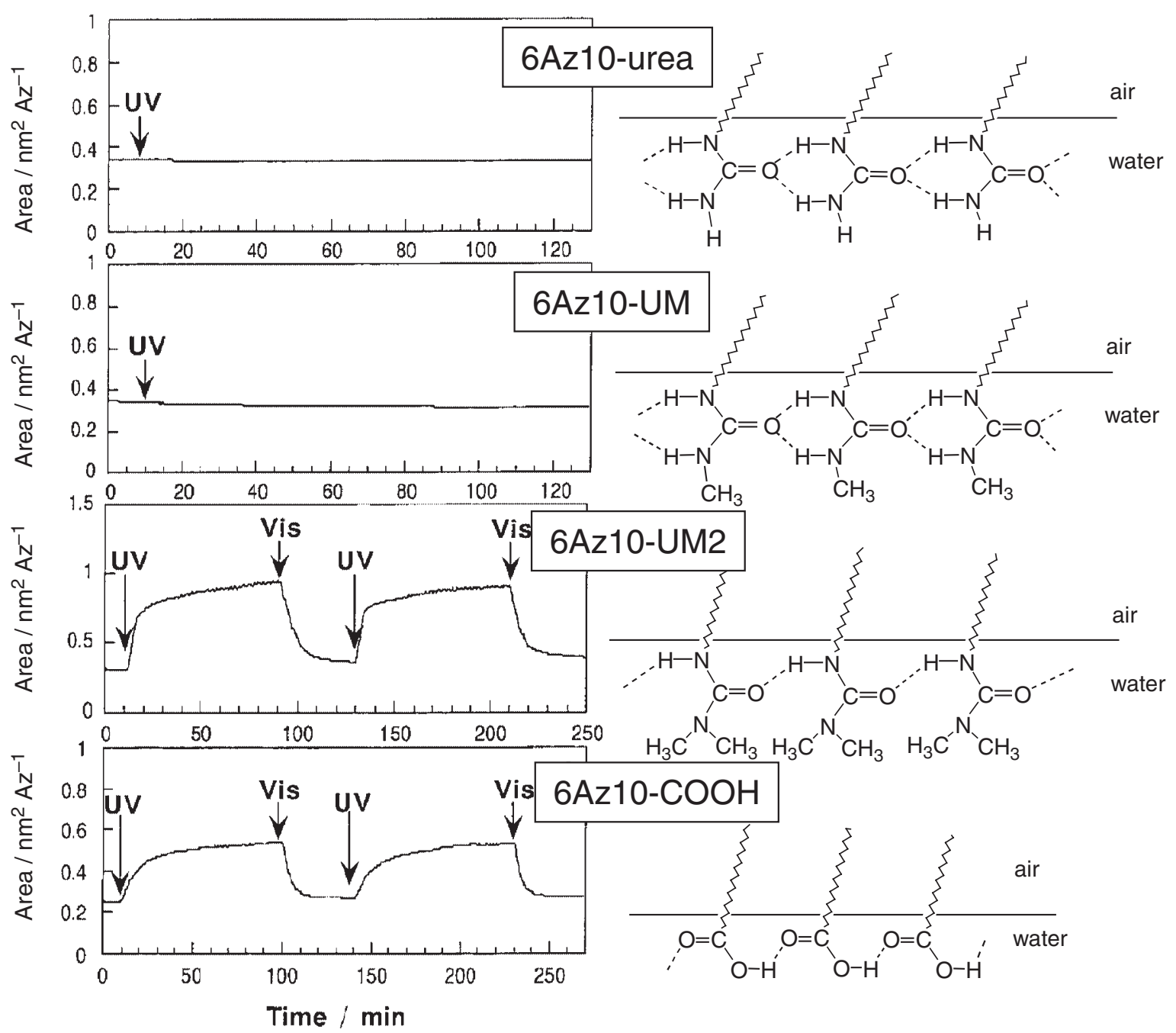

Figure 7. Photomechanical response of monolayers of three Az-urea derivatives and 6Az10-COOH (left hand side), and supposed model of hydrogen bonding formation on water (right hand side). Note that the photoresponse of monolayer is completely hindered for amphiphiles capable of forming the bifurcated hydrogen bonds (6Az10-urea and 6Az10-UM).
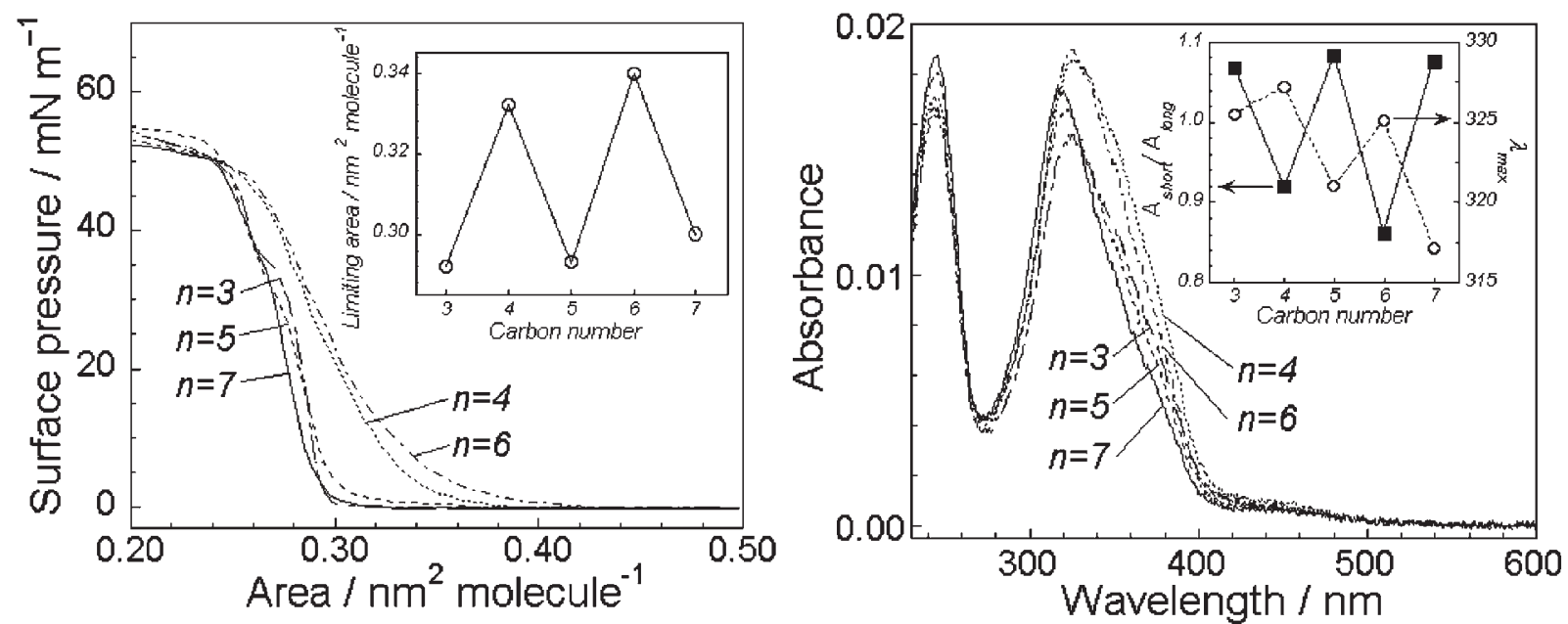

Figure 8. Odd-even effects observed for monolayers of 6Azn-urea derivatives ( $n=3-7)$. Surface pressure-area isotherms and limiting area per molecule (left and side), and UV-vis absorption spectra of 6Azn-urea monolayers transferred onto a quartz plate (right hand side). For the insets in the figures, see the text. 
A vast amount of investigations on photoalignment of LCs have been reported during the last one and half decades. Irradiation with linearly polarized light (LPL) induces photoinduced anisotropy in which molecules are generally oriented to an unexcitable direction, namely, perpendicular to the electric vector of the polarization direction. This effect is called the photoinduced optical anisotropy., ${ }^{1,4,5,72}$ Such photocontrol becomes particular effective in relatively viscous molecular organized media such as polymeric LCs $^{77-79}$ and LB films. ${ }^{80,81}$ Optically induced surface anisotropy in the Az polymer films has been successfully transferred to the orientation of various types of LC materials, as widely investigated by Ichimura et al., including thermotropic chiral nematic ${ }^{82}$ and discotic LCs ${ }^{83}$ lyotropic LCs, ${ }^{84}$ and also side chain liquid crystalline polymers. ${ }^{85}$

Exposure of the 6Az10-PVA monolayer to LPL induces the in-plane orientation of Az side chain as stated above. A spincast film of PDHS is subsequently prepared onto this photooriented Az monolayer. After crystallization the PDHS film exhibits a strong inplane optical anisotropy as shown in Figure $10 .^{73}$ The polarized absorption spectra reveals that the $\mathrm{Si}$ backbone is highly aligned perpendicular to the polarization plane of the actinic light. The aligned direction of PDHS main chain agrees with that of the Az orientation on the substrate. The crystallization of PDHS chain occurs on the photooriented $\mathrm{Az}$ monolayer in an epitaxial manner.

As expected, this phenomenon depends on the thickness of PDHS film. The orientational order of the PDHS backbone becomes higher for the thinner films. The effective control is available for films thinner than $c a .30 \mathrm{~nm},{ }^{74}$ which coincides with the region of so-called ultrathin films where the thickness is comparable to the coil dimension of a single polymer chain. ${ }^{86}$ When the molecular weight is in the range of ten thousand, the order parameter is enhanced after

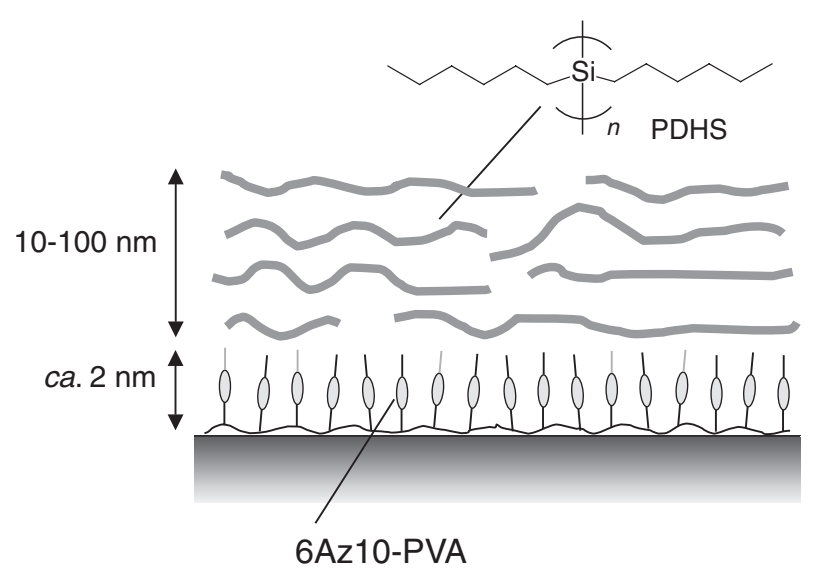

Figure 9. Schematic illustration of a PDHS cast film prepared on a 6Az10-PVA monolayer. another cycle of annealing and crystallization. For PDHS with molecular weight in the range of billions, the photoalignment is largely suppressed.

The length of tail part is anticipated to influence the order of photoinduced anisotropy because the $\mathrm{Az}$ monolayer is anchored to the hydrophilic substrate surface via the polar PVA backbone and the tail part is positioned to the outermost surface. Thus, PDHS should interact directly with the tail part of the Az side chain. In this motivation, the tail length (carbon number) is varied from 2 to $12 .{ }^{75}$ The photoinduced dichroic ratio (DR) of Az monolayers and the order parameter of PDHS give the largest values in the $\mathrm{Az}$ monolayer having $\mathrm{C}_{8}$ tail. The magnitude of the DR has a good relationship with the degree of spectral shift. Also, the 2D density of $\mathrm{Az}$ side chain in the monolayer is altered from 1.2 to $0.3 \mathrm{~nm}^{2}$ per Az unit. When the occupying area is $0.4 \mathrm{~nm}^{2}$ per Az unit, the largest anisotropy is obtained. In this optimum density, effective collective and cooperative motions as observed in LC materials are anticipated.

Figure 11 shows a polarized optical microscopic image of a locally photoaligned PDHS film indicated as a bent line. The rotation of the crossed polarizers indicates the alternated emergence and disappearance
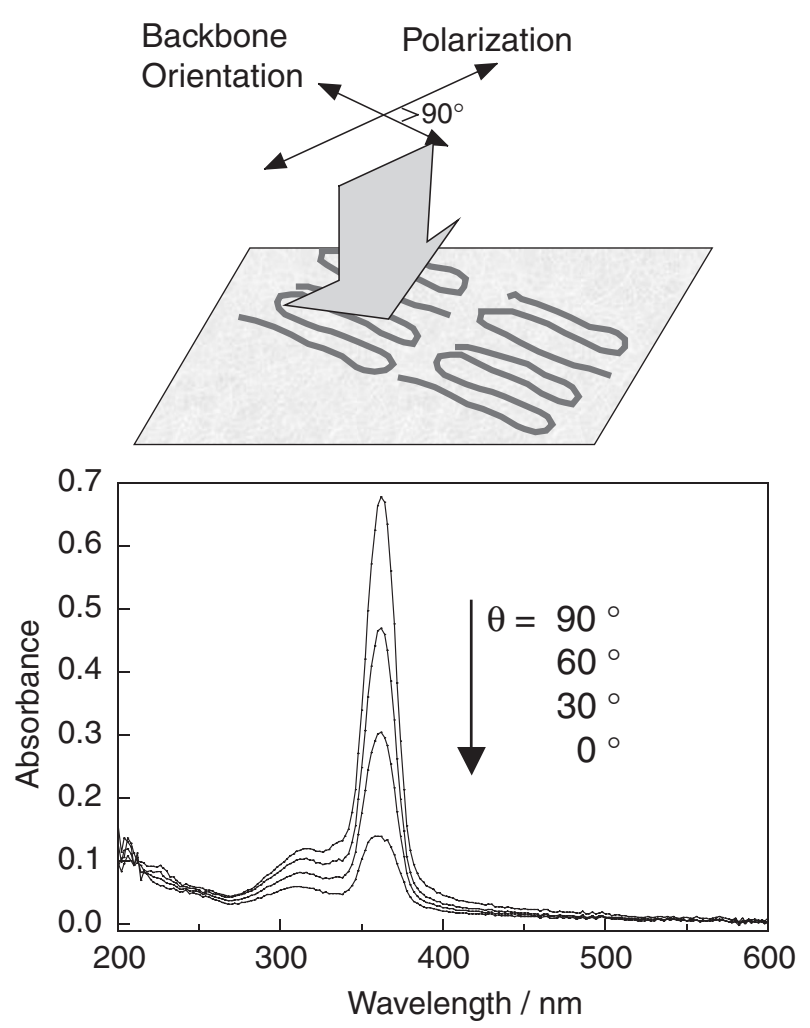

Figure 10. Polarized UV-vis absorption spectra of a PDHS film placed on a 6Az10-PVA monolayer pre-irradiated with polarized light. The backbone of PDHS is aligned perpendicular to the actinic polarized light. The upper illustration shows the geometric relationship between the light polarization and resulting polymer orientation. 
of the line at every $45^{\circ}$. This fact indicates that the bright line area is birefringent and the PDHS chains are uniaxially aligned. The dark parts are always dark by rotation, indicative of the random orientation of the polymer at micrometer levels. Thus, the micro-patterning of polymer alignment at $6 \mu \mathrm{m}$ resolution can be successfully achieved. ${ }^{75}$ In the photoprocess, basically any patterned shape is applicable. It would be impossible or laborious to attain such locally addressed orientation via conventional mechanical processes. This is a great benefit to apply photoprocess for polymer chain orientation.

\section{Photo-orientation of Mesoporous Silica Film}

Further challenge has been made for orientation of mesochannels of inorganic materials which are synthesized through the sol-gel condensation in the presence of templates of rod micelle aggregates of surfactant. ${ }^{87,88}$ Mesoporous materials are usually obtained as powders. However, recent efforts have been made to prepare thin films. Among them, attracting work has been reported by Miyata and Kuroda. ${ }^{89,90}$ They proposed the uniaxial alignment of mesoporous silica film deposited on a rubbed polyimide ${ }^{89}$ or an LB film..$^{90}$ If such surface-mediated alignment is applicable also by means of a photochemical process, one can expect a facile achievement of micro-patterning of alignment of mesochannels.

Such approach has been conducted by Kawashima et al. ${ }^{91,92}$ In this attempt, the photoorientation of the $\mathrm{Az}$ monolayer is first transferred to a photoorientated spincast film of PDHS as mentioned above, and then deposition of mesoporous silica film is performed (Figure 12).

In the optical microscopic images of the deposited surfactant/silica hybrids, uniaxially aligned elongated particles with widths of a micrometer range are observed. The orientation of each particle is highly

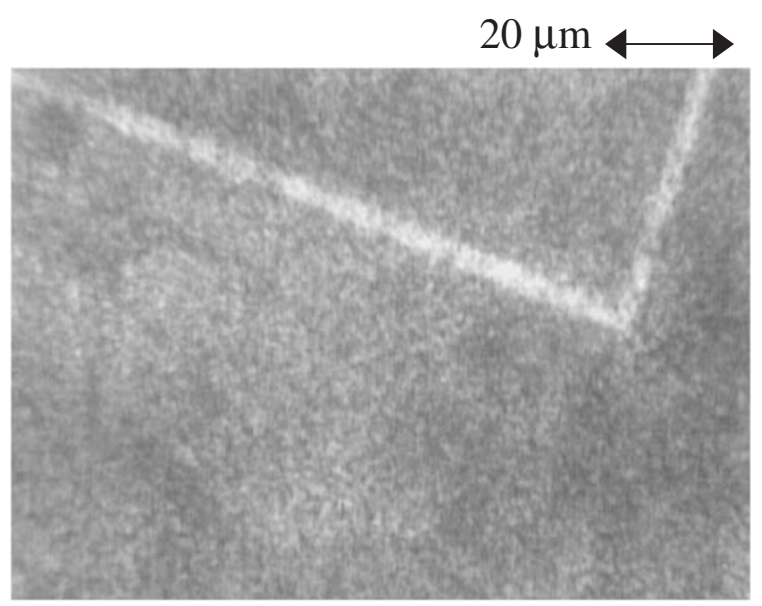

Figure 11. Locally aligned PDHS film irradiated with polarized light through a photomask. aligned parallel to each other, the direction of the longer side of silica particles being parallel to the polarization plane of the actinic LPL. A PDHS film surface without exposure to LPL yields round and spherical silica particles with no preferential orientation. The effect of photo-irradiation on the morphology of the deposited silica is obvious.

The patterning of mesochannel orientation can be performed as follows. ${ }^{92}$ A substrate modified with the 6Az10-PVA monolayer was first uniaxially photoaligned in the whole area as described above. Half of the area is then shadowed by a mask. The open area of the substrate is subjected to irradiation of LPL set in the polarization rotated by $90^{\circ}$. A spincast film of the PDHS is then prepared on this substrate with the $\mathrm{Az}$ monolayer. Figure 13 shows the optical microscopic image of the mesostructured surfactant/silica film deposited onto the above patterned surface. The underlying Si main chain in the two areas are perpendicular to each other, and the morphological features of the deposited mesostructured surfactant/silica hybrids characterized by formation of linear cracks are also perpendicular to each other accordingly. Such morphological feature strongly suggests the existence of aligned mesochannels existing in the distinct two directions.

Mesoporous silica can be obtained after removal of the organic surfactant template from the composite. ${ }^{91}$ The surfactant molecules are generally removed by calcination by heating up to high temperatures exceeding $573 \mathrm{~K}$. Here, photo-decomposition (photocalcination) by vacuum UV light achievable at room temperature is applied according to a modified method of Hozumi et al. ${ }^{93}$ It is expected that the distortion of the mesoporous structure in this method is more suppressed than that obtained via decomposition by heating.

The complete removal of the organic template surfactant can be confirmed by Fourier transform infrared spectroscopy for a photoaligned composite film synthesized on a silicon wafer. ${ }^{92}$ Before the photo-decomposition of organic components, the symmetric and asymmetric bands of $\mathrm{CH}_{2}$ and $\mathrm{CH}_{3}$ stretching are observed in the range of $2840-2970 \mathrm{~cm}^{-1}$. These bands are attributed to the alkyl chains in both surfactant molecule and PDHS. After photo-decomposition, no peaks are observed in these regions. The complete disappearance of these IR peaks indicates the removal of the "alignment template" of the PDHS film lying underneath is attained in addition to the template of the mesopores (surfactant molecules). On the other hand, the $\mathrm{Si}-\mathrm{O}$ stretching band around $1070 \mathrm{~cm}^{-1}$ is essentially unchanged, indicative of the retention of siloxane network structure. The removal of PDHS can be further confirmed by the disappearance of the 

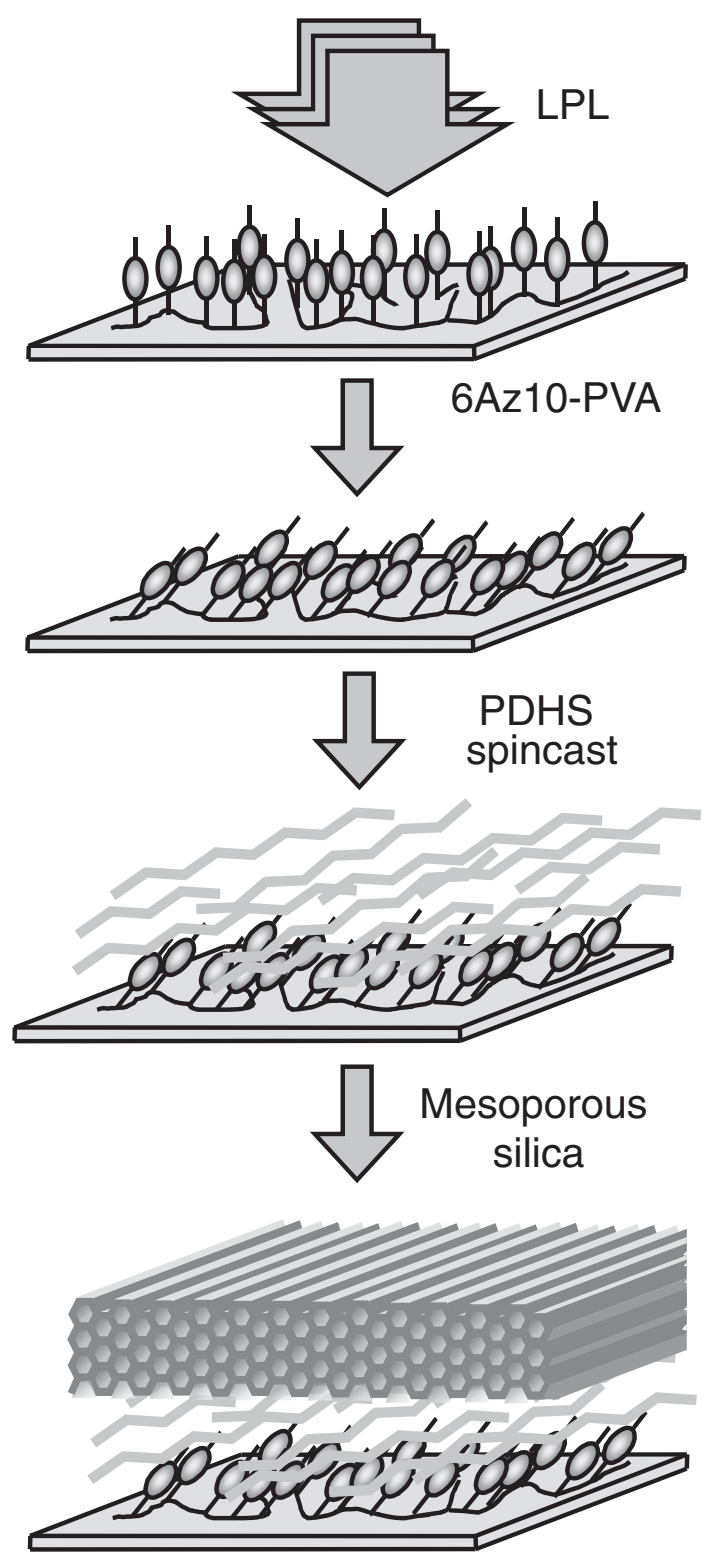

Figure 12. A scheme for photo-orientation of mesoporous silica film.

UV absorption band around $370 \mathrm{~nm}$.

The uniaxially aligned honeycomb mesochannel structure can be justified from X-Ray diffraction (XRD) patterns. ${ }^{91}$ In as-synthesized mesostructured surfactant/silica composite films, two diffraction peaks are observed at $2 \theta=2.63$ and 5.20 corresponding to (100) and (200) of the hexagonal packing of the mesochannels $\left(d_{100}=3.3 \mathrm{~nm}\right)$. The absence of the (110) and (210) diffraction peaks in this XRD pattern indicates that the mesochannels is oriented parallel to the PDHS surface plane. The in-plane orientation of the mesochannels can be further evaluated by the XRD measurement. The diffraction intensity at a grazing angle gives the maximum at every $180^{\circ}$ in the sample rotation, indicating that the photo-oriented mesochannels have a preferential orientation perpendicular to the direction of the polarization of the actin-

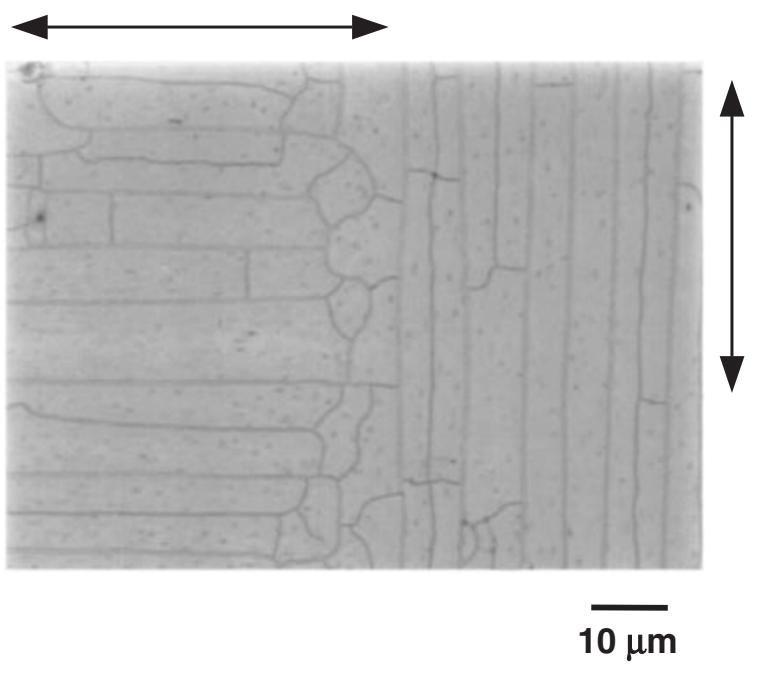

Figure 13. Optical microscopic image of a mesoporous silica film oriented with two polarized light beams. The arrows indicate the direction of the electric vector of the polarized light.

ic light over the whole film plane.

Decisive evidence for the formation of uniaxially aligned mesochannels is obtained by transmission electron microscopy (TEM). ${ }^{92}$ The two TEM photographs in Figure 14 depict the cross-sectional structure of the as-synthesized surfactant/silica hybrid film observed in the two directions orthogonal to each other. Figure 14 (upper) shows the image when the film is sliced parallel to the direction of the actinic polarized light. The hexagonal structure of the mesochannels is clearly visualized through overall thickness of the film. On the other hand, when the film is sliced perpendicular to the direction of the actinic polarized light, the lines with a periodicity of 3-4 nm running parallel to the substrate are observed (Figure 14 (lower)). The periodic length coincides well with that of the hexagonal spacing obtained by XRD. The cylindrical structure of the mesochannels is fully stretched straight in the film. Figure 14 (lower) obviously visualizes the hetero-interface regions composed of the $\mathrm{Si}$ wafer (the dark region at the bottom), Az monolayer ( $2 \mathrm{~nm}$ thickness, not clearly shown), PDHS film (40$60 \mathrm{~nm}$ thickness, brighter region) and deposited mesochannels with parallel stripes.

\section{PHOTO-TRIGGERED INSTANT MASS MIGRATION}

This section describes another attracting phenomenon, instant photo-triggered mass migration, exhibited as a consequence of a strong and dynamic molecular cooperativity. Here, the motions involved are not at a monolayer level but at collective mass levels in thin films. The films of typically less than $100 \mathrm{~nm}$ thickness are prepared by simple spin-casting. Here, the amphiphilic and liquid crystalline polymers spon- 

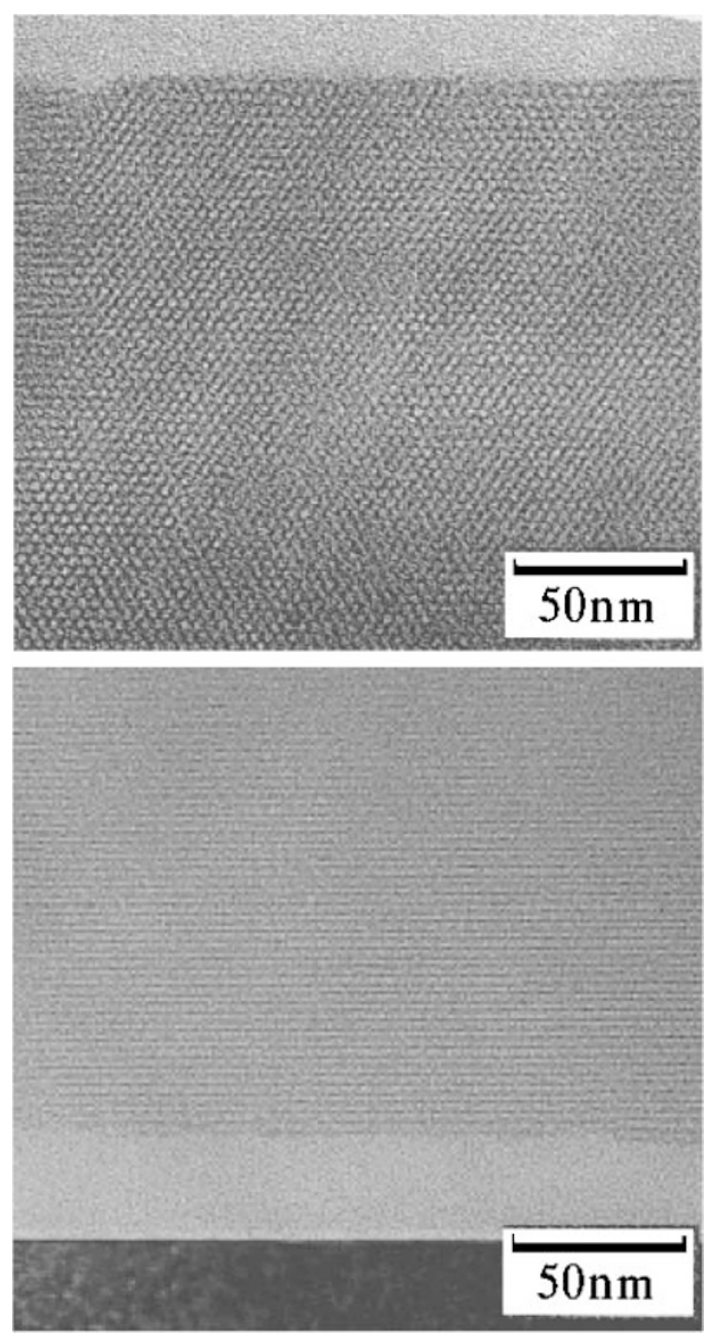

Figure 14. TEM images of a mesoporous silica film sliced in different directions; sliced in parallel (upper) and perpendicular (lower) to the polarized light. The aligned hexagonal packing of the photoaligned mesochannels is clearly observed.

taneously forms organized smectic bilayer structures within the film. In this sense, the phenomena observed have close connections with data obtained with monolayer systems.

Azobenzene polymers are potentially useful as materials for reversible holographic information storage and photonic devices. ${ }^{94-98}$ Surface relief grating (SRG, regular topological surface modification) formed via the irradiation with an interference pattern of coherent light has been demonstrated recently ${ }^{99-101}$ and is perhaps the most attracting target in the current research of Az polymers. A great deal of data has been accumulated rapidly due to its basic phenomenological interest ${ }^{102-108}$ and to attractive technological applications. ${ }^{106,108}$ This process has particular technological advantages in view of relief formation since i) it offers a facile, all-optical and single step fabrication process that does not require a wet development procedure, and ii) the surface topology is erasable by application of circularly polarized light or heating above glass transition temperature $\left(T_{\mathrm{g}}\right)$, which realizes the repeatable utilization. It is of no doubt that the SRG is formed via large-scale polymer chain migration, however, the precise mechanism is still the subject of intensive investigation.

Earlier works are mostly devoted to inscription of gratings using interference light beams, thus the phenomenon has frequently been called as SRG. However, the mass migration and relief formation is not limited to formation of gratings, namely, irradiation though a patterned photomask or a single beam also induces migration as will be indicated below. The term "photoinduced surface relief (PSR)" seems more appropriate to express these processes as proposed by Fukuda. ${ }^{109}$ Typical Az-containing polymers used for these studies are indicated in Figure 15.

\section{Binary Component Systems}

Unique photoinduced mass migration systems are proposed by Ubukata et al. using liquid crystalline binary component materials comprised of 6Az10-PVA and 5CB (see Figure 15). ${ }^{110-112}$ This work was initiated as a serendipitous extension during evaluations of the command surface model using 6Az10-PVA/ 5CB hybrid LB films. ${ }^{51-53,113}$ The hybrid films are irradiated with non-polarized UV $(365 \mathrm{~nm})$ light in advance to attain a cis-rich photoequilibrated state (UV light treatment). Starting from this state, the irradiation that induces the film migration is performed by argon ion laser beam $(488 \mathrm{~nm})$ which also induces back reaction to the trans form. The growth of the first-order diffraction efficiency monitored with a $\mathrm{He}-\mathrm{Ne}$ laser and morphological evaluation by AFM indicate that the mass migration is attained at very small dose levels as less than $100 \mathrm{~mJ} \mathrm{~cm}^{-2}$, which is comparable to photopolymer systems for photoresists. Here, the energy level is three orders of magnitude smaller than those required for conventional amorphous polymer systems hitherto reported. This means that the process which formerly required several ten minutes illumination can be shortened to that of second levels with a beam of identical intensity.

Figure 16 indicates an essential feature of this binary system. This figure displays the diffraction efficiency (closed circles) and the surface modulation depth (open circles, $\Delta h$ ) of the hybrid films at various molar fractions performed after UV light treatment. The diffraction efficiency shows a sudden increase at $f=0.67$, which corresponds to the stoichiometry of two 5CB molecules per one Az unit. Below and above this ratio the diffraction efficiency rapidly decreases. The profile of $\Delta h$ almost follows that of the diffraction efficiency to give the maximum depth $(100 \mathrm{~nm})$ at $f=0.67$. Differential scanning calorimetric (DSC) analysis for the bulk mixtures ${ }^{11}$ and the Lang- 
<smiles>CCCCCCCCN(CC)c1ccc(N=Nc2ccc([N+](=O)[O-])cc2)cc1</smiles>

6

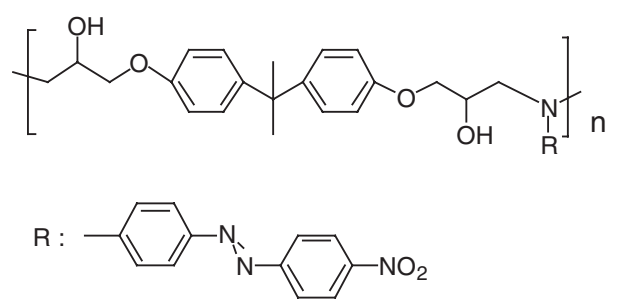

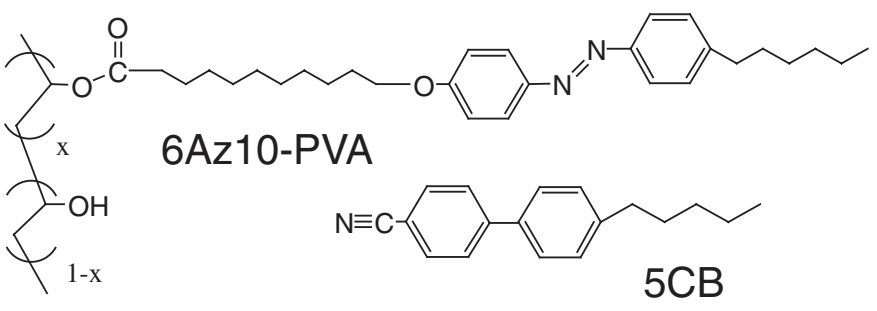

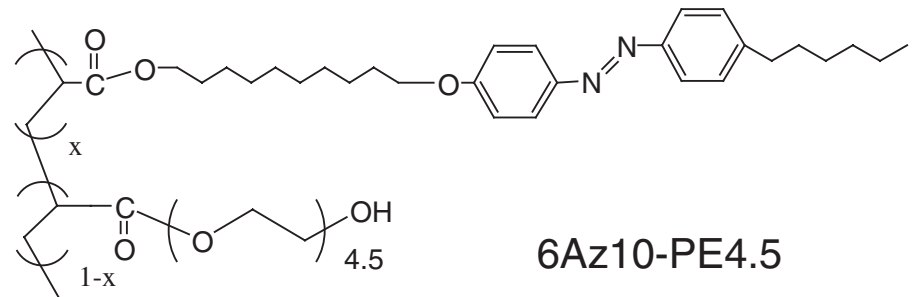

7

Figure 15. Typical Az-containing polymers used for studies of photoinduced mass transfer.

muir monolayer experiments on water ${ }^{53}$ both show that one 6Az10-side chain can accommodate two $5 \mathrm{CB}$ molecules. Therefore, $5 \mathrm{CB}$ acts not as only a plasticizer but provides the stoichiometric supramolecular material. The instant mass migration is the result from the cooperative interplay between 6Az10PVA and 5CB.

\section{Soft Crosslinkable Polymers}

Another important requirement for relief forming material is the shape stability in terms of long-term storage, and durability against water, organic vapors and heating. The stability can be improved when one employs amorphous polymers with high $T_{\mathrm{g}}{ }^{114,115}$ or liquid crystalline polymers having high $T_{\mathrm{i}}$ (transition temperature to isotropic state). ${ }^{116}$ However, high $T_{\mathrm{g}}$ or $T_{\mathrm{i}}$ materials severely reduce the mass mobility.

Zettsu et al. have developed unique soft and crosslinkable liquid crystalline polymers. ${ }^{117,118}$ The polymer involves an oligo(ethylene oxide) (EO) side chain (6Az10-PE4.5, see Figure 15). Introduction of the EO chain gives the effects similar to the incorporation of low-molecular-mass LC molecules, and the instant mass migration also occurs. After the surface relief structure is formed, the polymer is then subjected to chemical crosslinking via formalization (acetal formation with formaldehyde) between the hydroxyl groups at the terminus in the EO chain. AFM observations justify the remarkable stability improvement after crosslinking (Figure 17). The SRG structure disappears by heating at $80^{\circ} \mathrm{C}$ for the untreated film whereas the formalized film exactly preserves the surface modulation pattern even at $250^{\circ} \mathrm{C}$ without any damage. The process employed here can be compared with a simple approach using $\mathrm{Az}$ polymers of high $T_{\mathrm{g}}$. Fukuda et al. ${ }^{114,115}$ employed maleimide-based

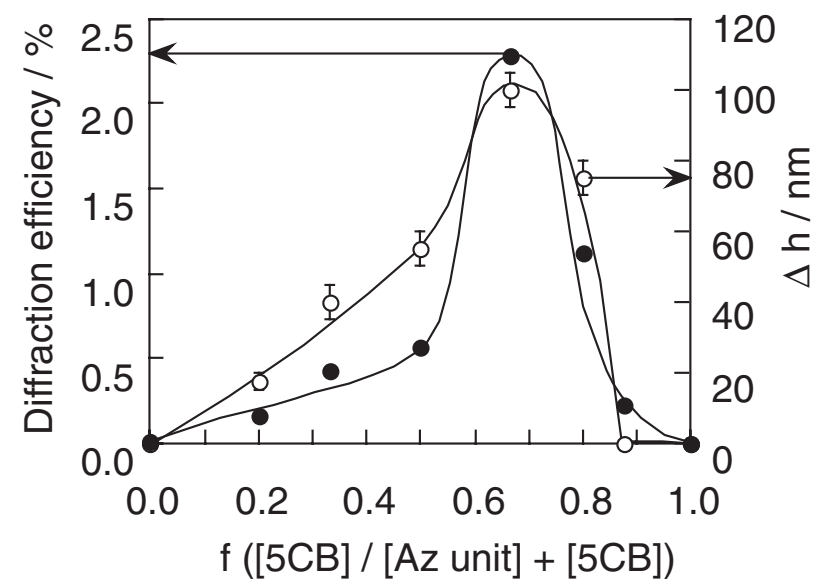

Figure 16. Diffraction efficiency and top-to-valley height of the relief gratings inscribed by two interference $\mathrm{Ar}$ ion laser beams as a function of $5 \mathrm{CB}$ fraction of the $5 \mathrm{CB} / 6 \mathrm{Az} 10-\mathrm{PVA}$ mixtures. Note that the mass transfer becomes prominent at the $1: 2 \mathrm{Az} / 5 \mathrm{CB}$ ratio.

high $T_{\mathrm{g}}$ amorphous polymer $\left(T_{\mathrm{g}}=170-279^{\circ} \mathrm{C}\right)$. In their polymer systems, the thermal stability is considerably improved, however, in compensation for requirement of vast amounts of exposure energy. Light doses required for SRG inscription for such high $T_{\mathrm{g}}$ polymer typically range some hundred $\mathrm{J} \mathrm{cm}^{-2}$. The soft and crosslinked 6Az10-PE4.5 realizes unique strategy in which the mass migration is achieved in the soft state and immobilized at any desired stage providing marked thermal stability comparable with high $T_{\mathrm{g}}$ polymers.

\section{On the Mechanism of Instant Mass Migration}

The mass migrating systems mentioned here has no polarization dependence, which shows marked contrast with widely known properties for hitherto inves- 

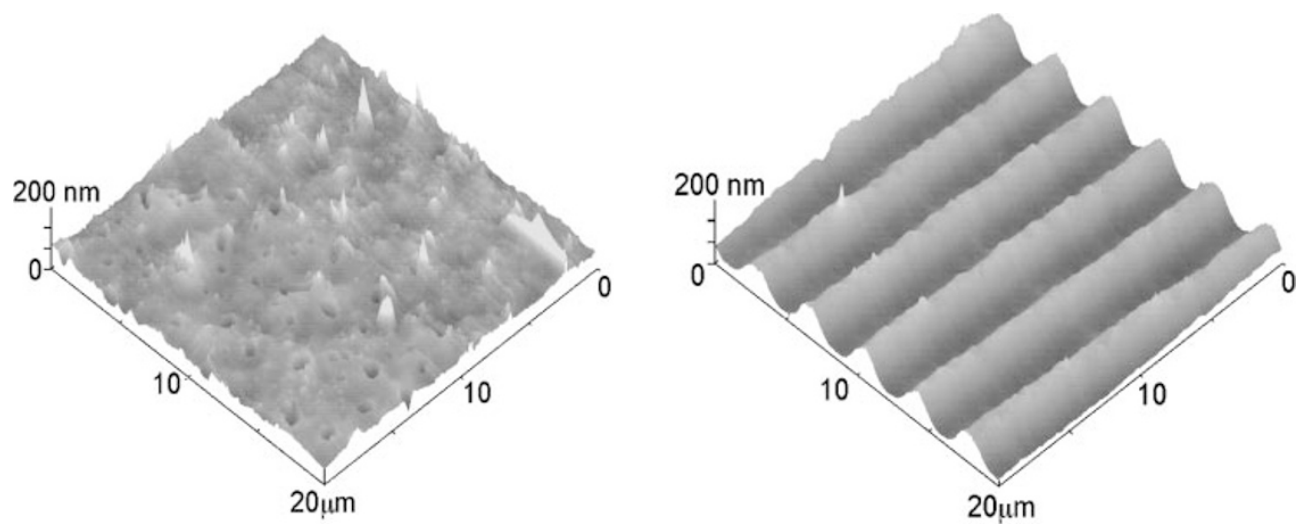

Figure 17. AFM images demonstrating the shape stability against heating. Inscription of relief structure is first performed on a 6Az10PE4.5 film. Without crosslinking, the structure is readily destroyed after heating at $100{ }^{\circ} \mathrm{C}$ (left). In contrast the shape is fully maintained after heating at $250{ }^{\circ} \mathrm{C}$ after the crosslinking is performed.

tigated polymers. In general, the migration shows strong polarization dependency, and the migration proceeds in the direction of electric field of light. ${ }^{106-108}$ The absence of polarization dependency in the instant mass migration systems can be justified at least from two kinds of experiments, a double beam exposure experiment varying the polarizations configurations and irradiation through a patterned photomask with non-polarized light.

Figure 18 shows the growth of the first order diffraction intensity due to SRG formation starting from the cis-rich Az polymer film as a function of irradiation time under different polarization combinations. Irradiation experiments are carried out under three kinds of polarization configurations $[(s-: s-),(p-: p-)$, $(s-: p-)]$. When the two parallel writing beams are irradiated in intensity grating conditions, i.e., $(s-: s-; \bigcirc)$ or $(p-: p-; \square)$ having no spatial variation of polarization (intensity hologram), the SRGs are readily formed. There is essentially no dependence of the SRG inscription rate under the two intensity holographic conditions. On the other hand, when the two interfering beams are orthogonal polarized $(s-: p-; \diamond)$, providing the pure polarization recording condition (polarization holography), no growth of the diffraction intensity profile is observed.

The lack of polarization dependence leads to a validity of the process using a photmask and a non-polarized light from a mercury lamp. Figure 19 shows a photomask with a grid period of $4 \mu \mathrm{m}$ (transmitting window) $\times 2 \mu \mathrm{m}$ (shadow) and the resulting relief structure. In this case, there is no optical-field component present in any in-plane direction. The features of the surface topology evidently indicate that the mass migration occurs from the illuminated regions to the unilluminated ones within the plane. The relief structure has two distinct heights. The corners have nearly the double height of the sides of the square windows.

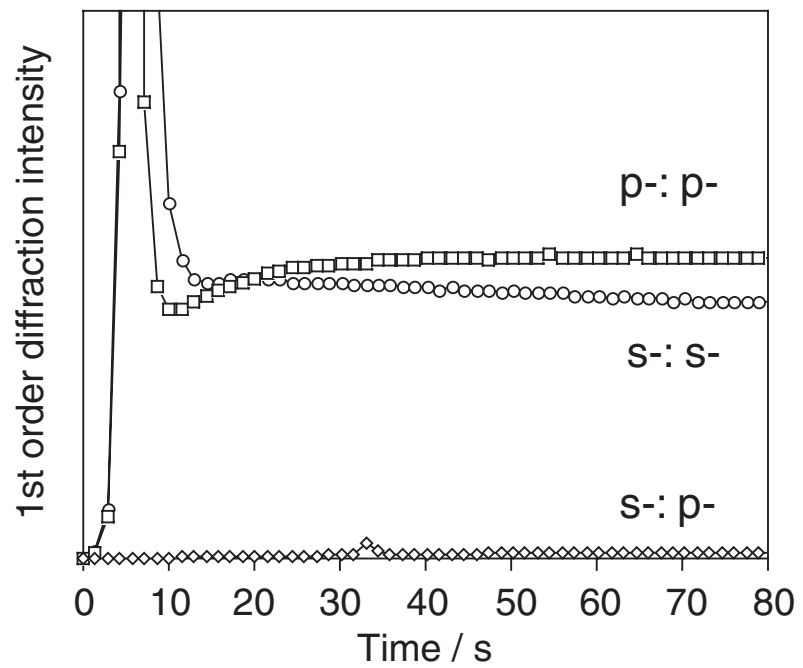

Figure 18. Growth of diffraction efficiency by illumination with two polarized light beams. These data show that only the intensity modulation induced the mass transfer and the polarization dependency is negligible.

This fact strongly suggests that the mass migration occurred evenly within the plane, namely, the film mass coming from four directions (corners) has the double height of that from two directions (sides). It is stressed that, when the thickness of the initial film is less than $100 \mathrm{~nm}$, the film material is almost perfectly expelled from the illuminated area under suitable conditions.

Unexpectedly, mass transfer in the hybrid film continued proceeding even in the dark after switching off the light irradiation. ${ }^{119,120}$ Figure 20 shows the selfgrowth behavior of the shallow cylindrical holes produced by spot irradiation (diameter, $20 \mu \mathrm{m}$ ) with a white-light beam to the hybrid film. The bright circle in the optical microscopic image is a hole and the dark annulus is a thicker rim. As shown, the cylindrical hole spontaneously grew in size with time. Selfgrowth of the hole continues for approximately $6 \mathrm{~h}$, 

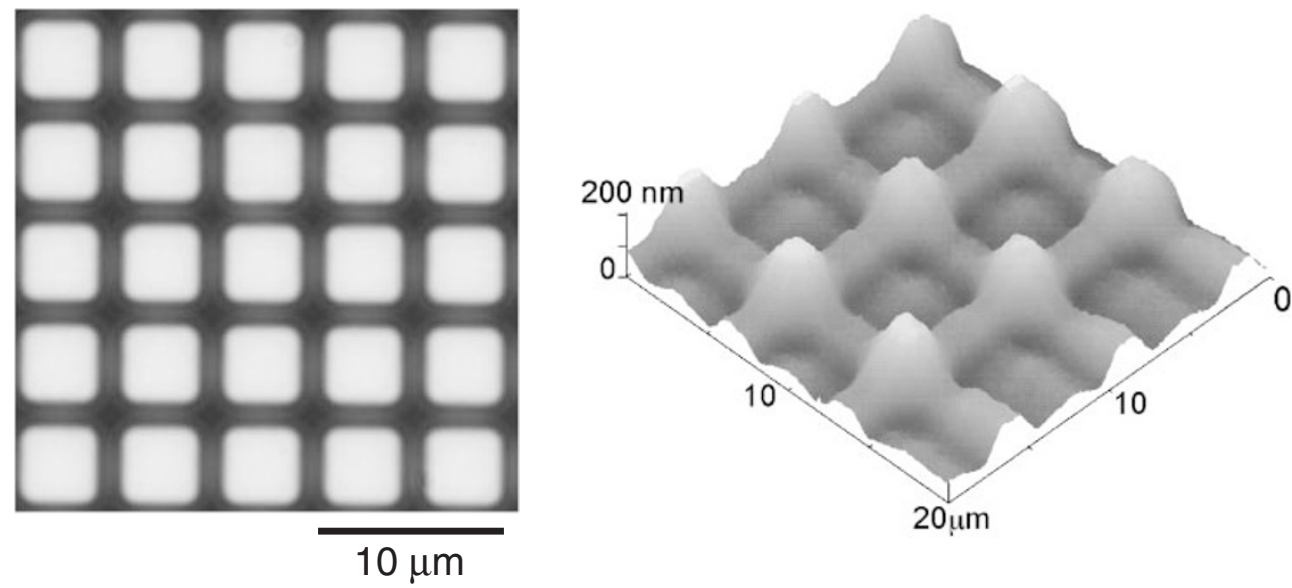

Figure 19. Inscription of relief structure by illumination through a grid type photomask. The photomask (left) and the resulting relief structure (right).

creating a hole having an approximately three-fold larger diameter than the initial one. Such a spontaneous thermal process should be related to dewetting observed in thin fluid polymer films.

The lack of polarization dependence and migration in the dark clearly indicate that the processes involved in this instant mass migration are thermal in essence. The thermal driving factors can involve an attractive force that may work between the isotropic and liquid crystalline phase, surface tension, dewetting etc. In this context, the process dealt with here should be considered as "photo-triggered" thermal migration rather than "photo-induced" one.

\section{Conveyer Actions}

Thus far, applications of photoinduced migrations considered have been almost limited to utilizations of resulting static relief structures. They involve holographic recording, phase mask, LC alignment, waveguide couplers, fabrication of intricate surfaces etc. ${ }^{1,106,108}$ In the instant mass migrating system, the motion itself can be a valuable function. In this section application as "molecular conveyer" for patterning of light-inert (non-photoresponsive) functional materials is proposed. ${ }^{119}$ Functional materials such as dye molecules, conjugated polymers, and semiconductor quantum dots can be conveyed to the massive motion of the Az polymers.

A blue rodlike diaminoanthraquinone-type dye is embedded into the hybrid film of 6Az10-PVA and $5 \mathrm{CB}$. At higher dye content, e.g. $10 \mathrm{~mol} \%$ of the LC molecule, the UV irradiation followed by dark adaptation at room temperature for a day leads to a growth of dendritic crystals of the dye (Figure 21 top). When the crystallization is performed after the photoinscription of the lined relief ( $18 \mu \mathrm{m}$ width in this case), the direction of dye organization is restrained and aligned along the relief structure (middle). After sufficient
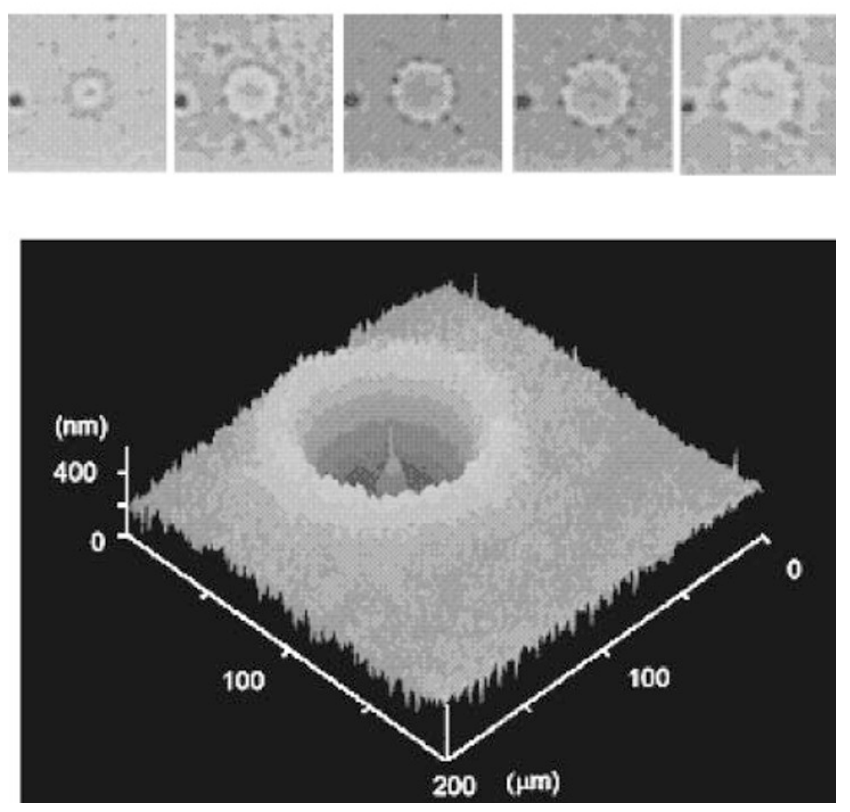

Figure 20. Continuing mass transfer in the dark after irradiation with a light beam of $20 \mu \mathrm{m}$ diameter. Optical microscopic images (upper) and AFM image after $4 \mathrm{~h}$ (lower) are shown.

crystallization at room temperature, the protruding line showed high optical anisotropy. The polarized absorption spectra (bottom) reveal that the dichroic dye is aligned in the direction of the inscribed line. As shown, the absorption moment of the dichroic dye was highly aligned parallel to the direction of the inscribed line. Most probably, the orientational anisotropy is induced by a uniaxial growth in the diffusionlimited crystallization restricted in the one dimensional geometry. ${ }^{119}$

Application of the mass conveying principle is not limited to low-molecular-mass molecules; a larger material, semiconductor nanocrystals $(\mathrm{NCs})^{121}$ of 
$6 \mathrm{~nm}$ diameter with $6 \times 10^{5}$ mass weight can be also conveyed. ${ }^{119}$ Spin-cast films are prepared from mixed solution composed of 6Az10-PVA, 5CB, and cadmium selenide (CdSe) NCs. After a short light exposure through a grating photomask, surface relief undulations are clearly formed. Observation of luminescence from NCs by fluorescence microscopy shows that NCs are transported following the patterned irradiation.

The conveying action may be widely applicable to many other kinds of functional materials that are themselves inert to light. A great advantage to use the instant migration system can be the requirement of small amounts of light, which would not damage the host materials.

\section{CONCLUSIONS}

Many years have been passed since 1937 when the photoisomerization of the $\mathrm{Az}$ dye was discovered. ${ }^{122,123}$ It seems, nevertheless, that attempts for cre-
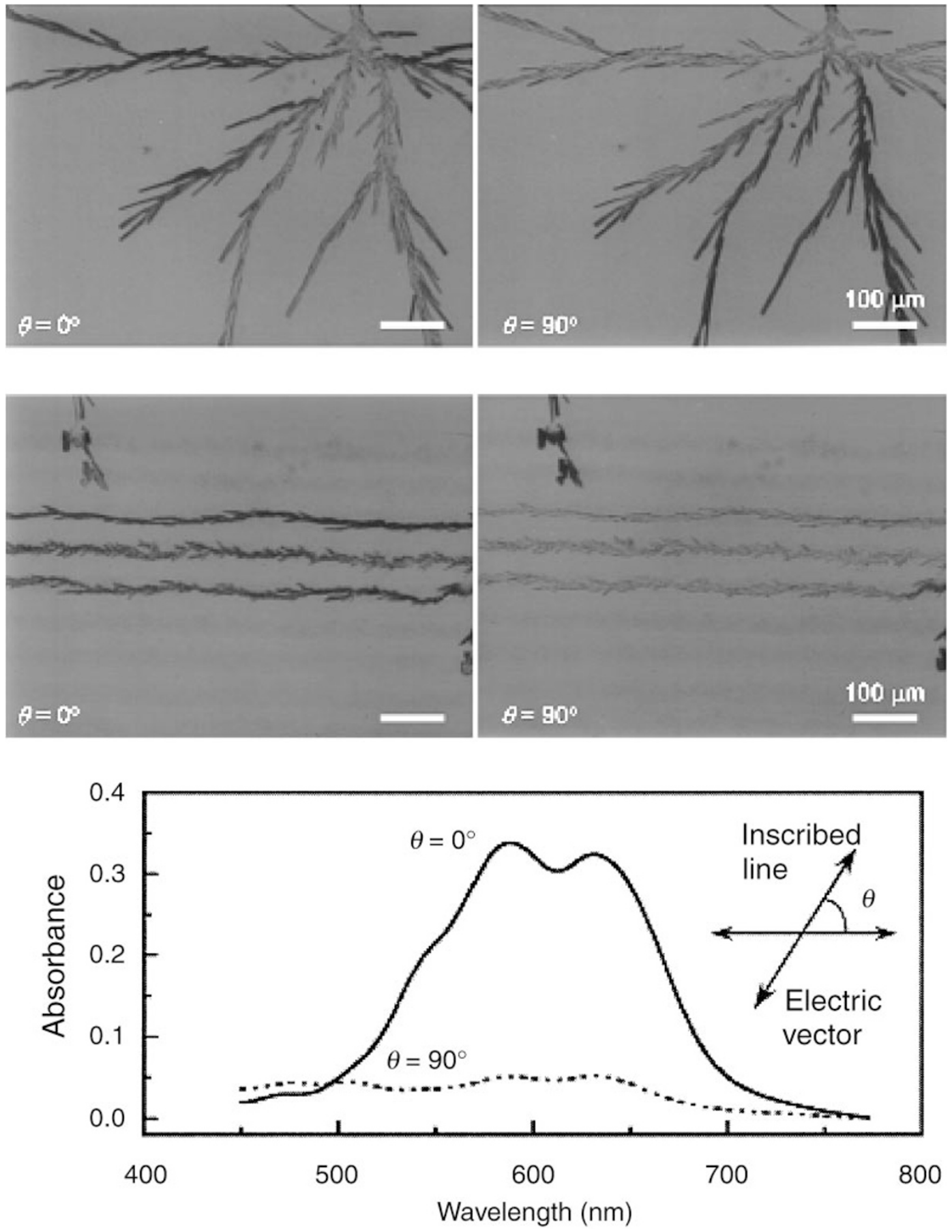

Figure 21. Crystallization of an anthraquinone type dye on a 6Az10-PVA/5CB film after UV light illumination without relief inscription (top) and after line relief inscription (middle). The polarized absorption spectra taken for the middle are shown in the bottom. Note that highly molecular alignment is attained as a consequence of one dimensional dye diffusion in the crystallization process. 
ation of new functionalities using Az polymers is still expanding probably due to strong motivations mentioned in the introductory remarks. Azobenzene and also other types of photochromic unit can be regarded as a light-driven sub-nano machine. Here, how we can transfer or amplify this molecular motion to larger hierarchies in the materials becomes a very important scenario. In this sense, design of flexible molecular organizations where sufficient motional freedom is allowed becomes important. The approach focusing onto monolayers and comparable films summarized in this article seems most effective and reasonable. This article overviewed photomechanical effects in monolayers, orientational transfer of photooriented monolayers to other anisotropic materials, and finally light-driven instant massive motions of organized Az-containing polymers. The significance of polymer organization and handling at interfaces is not limited to interests in fabrication of light-responsive systems. We anticipate that such strategy will provide many implications in creations of various kinds of stimulus or environment-responsive functions of soft materials.

This article introduced only limited topics and activities regarding monolayers or thin films thinner than $100 \mathrm{~nm}$. For thicker films ranging over micrometer level thickness, intensive studies are being undertaken for production of photonic materials and devices that can be used for holographic memories and light modulators. ${ }^{2,5,124}$ New developments in organized Az-containing polymer and LC systems in Japan are worth describing. Examples are as followings. With respect to photomechanical actuation, Ikeda et al. developed new LC elastomer films that show photoinduced bent or roll in response to light along the direction of rubbing ${ }^{125}$ or LPL electric vector. ${ }^{126}$ Furukawa et al. fabricated a solvent-swollen Az-containing fibrous gel that shows sharp bent by illumination. ${ }^{127}$ As light modulation systems, the structural color of a hydrogel obtained after templating of crystalline spheres ${ }^{128}$ can be switched by the photoisomerization of an embedded Az unit as shown by Takeoka et al. ${ }^{129}$ Research on light modulation and memory/rewriting effects in cholesteric LC systems are also making marked progresses as shown by Tamaoki et al. ${ }^{130}$ and Moriyama and Kato. ${ }^{131}$ It is emphasized that above new trends together with light-driven thin film systems described in this article strongly indicate the versatility and utility of Az-containing polymers as promising future materials.

Acknowledgment. The author thanks Prof. K. Ichimura for long collaboration and encouragement. Collaboration and discussions with Profs. T. Iyoda and M. Nakagawa, and Drs. S. Morino, K. Arimitsu, and Y. Hayashi are greatly appreciated. The author thanks co-workers, Mr. H. Ogasawara, Dr. T. Ubukata, Dr. S. Nagano, Mr. T. Fukuchi, Mr. J. Kojima, Dr. K. Fukuda, Dr. Y. Kawashima, Dr. T. Kobayashi, and Mr. N. Zettsu for their insightful contributions and efforts involved in this review. The author also thanks Profs. H. Yamaoka, H. Matsuoka, and Dr. T. Fukuda for valuable collaborations. The work has been supported by Grant-in-Aid for Scientific Research on Priority Areas from MEXT (No. 277, 404 and 417), Nissan Science Foundation, Shimadzu Science Foundation, and Asahi Glass Science Foundation.

\section{REFERENCES}

1. A. Natansohn and P. Rochon, Chem. Rev., 102, 4139 (2002).

2. "Photoreactive Organic Thin Films," Z. Sekkat and W. Knoll, Ed., Academic Press, San Diego, CA, 2002.

3. G. S. Kumar and D. C. Neckers, Chem. Rev., 89, 1915 (1989).

4. K. Ichimura, Chem. Rev., 100, 1847 (2000).

5. T. Ikeda, J. Mater. Chem., 13, 2037 (2003).

6. H. Rau, in "Photochemistry and Photophysics," J. F. Rebeck, Ed., 2, CRC Press, Boca Raton, FL, 1990, Chap 4, p 119.

7. H. Rau, "Photoisomerization of Azobenzenes," Chap 1 in reference 4.

8. T. Seki and K. Ichimura, Polym. Commun., 30, 108 (1989).

9. T. Seki and K. Ichimura, Thin Solid Films, 179, 77 (1989).

10. K. Nishiyama, M. Kurihara, and M. Fujihira, Thin Solid Films, 179, 477 (1989).

11. T. Seki, 'Photomechanical Responses in Photochromic Molecular Films and Relating Phenomena,' in 'Handbook of Photochemistry and Photophysics," M. S. A. AbdelMottaleb and H. S. Nalwa, Ed., American Scientific Publishers, Stevenson Ranch, CA, 2003, Vol 2, Chap 9, p 435.

12. T. Seki and K. Ichimura, "Dynamic Photocontrold of Molecular Organization and Motion of Materials by TwoDimensionally Arranged Azobenzene Assemblies," Chap 15 in reference 2.

13. M. Irie, Adv. Polym. Sci., 94, 27 (1990).

14. H. S. Blair, H. I. Pague, and J. E. Riordan, Polymer, 21, 1195 (1980).

15. H. S. Blair and C. B. McArdle, Polymer, 25, 1347 (1984).

16. B. R. Malcolm and O. Pieroni, Biopolymers, 29, 1121 (1990).

17. B. R. Malcolm, Thin Solid Films, 178, 17 (1989).

18. A. Ahluwalia, R. Piolanti, and D. De Rossi, Langmuir, 13, 5909 (1997).

19. O. N. Oliveira Jr., D. M. Taylor, and H. Morgan, Thin Solid Films, 210-211, 76 (1992).

20. H. Menzel, Macromol. Chem. Phys., 195, 3747 (1994).

21. H. Menzel and M. L. Hallensleben, Polym. Bull., 27, 89 (1991).

22. H. Menzel, Macromolecules, 26, 6226 (1993).

23. C. Steinem, A. Janshoff, M. S. Vollmer, and M. R. Ghadiri, Langmuir, 15, 3956 (1999).

24. M. S. Volmer, T. D. Clark, C. Steinem, and M. R. Ghadiri, 
Angew. Chem., Int. Ed., 38, 1598 (1999).

25. J. W. Weener and E. W. Meijer, Adv. Mater., 12, 741 (2000).

26. A. Shidorenko, C. Houphouet-Boigny, O. Villavicencio, M. Hashemzadeh, D. V. McGrath, and V. V. Tsukruk, Langmuir, 16, 10569 (2000).

27. T. Seki and T. Tamaki, Chem. Lett., 1993, 1739.

28. T. Seki, R. Fukuda, M. Yokoi, T. Tamaki, and K. Ichimura, Bull. Chem. Soc. Jpn., 69, 2375 (1996).

29. T. Seki, H. Sekizawa, R. Fukuda, T. Tamaki, M. Yokoi, and K. Ichimura, Polym. J., 28, 613 (1996).

30. K. Kago, M. Fuerst, H. Matsuoka, H. Yamaoka, and T. Seki, Langmuir, 15, 2237 (1999).

31. R. Wüstneck, V. B. Fainerman, and V. Zauls, J. Phys. Chem. B, 103, 3587 (1999).

32. R. Wüstneck, D. Prescher, S. Katholy, G. Knochenhauer, and L. Brehmer, Colloids Surf. A, 175, 83 (2000).

33. D. Y. Kwok, D. Vollhardt, R. Miller, D. Li, and A. W. Neumann, Colloids Surf. A, 88, 51 (1994).

34. D. Hönig and D. Möbius, J. Phys. Chem., 95, 4590 (1991).

35. S. Hénnon and J. Meunier, Rev. Sci. Instrum., 62, 936 (1991).

36. T. Seki, H. Sekizawa, and K. Ichimura, Polymer, 38, 725 (1997).

37. T. Seki, H. Sekizawa, S. Morino, and K. Ichimura, J. Phys. Chem. B, 102, 5313 (1998).

38. T. Seki, H. Sekizawa, and K. Ichimura, Polym. J., 31, 1079 (1999).

39. T. Seki, K. Tanaka, and K. Ichimura, Macromolecules, 30, 6401 (1997).

40. T. Seki, J. Kojima, and K. Ichimura, Macromolecules, 33, 2709 (2000).

41. D. Beaglehole, E. Z. Radlinska, B. W. Ninham, and H. K. Christenson, Phys. Rev. Lett., 66, 2048 (1991).

42. Y. L. Chen and J. N. Israelachivili, J. Phys. Chem., 96, 7752 (1992).

43. M. Matsumoto, D. Miyazaki, M. Tanaka, R. Azumi, E. Manda, Y. Kondo, N. Yoshino, and H. Tachibana, J. Am. Chem. Soc., 120, 1479 (1998).

44. M. Matsumoto, S. Terrettaz, and H. Tachibana, Adv. Colloid. Interface Sci., 87, 147 (2000).

45. T. Seki, J. Kojima, and K. Ichimura, J. Phys. Chem. B, 103, 10338 (1999).

46. K. Ebihara, S. Koshihara, M. Yoshimoto, T. Maeda, T. Ohnishi, H. Koinuma, and M. Fujiki, Jpn. J. Appl. Phys., 36, L1211 (1997).

47. J. Kumaki, Y. Nishikawa, and T. Hashimoto, J. Am. Chem. Soc., 118, 3321 (1996).

48. K. Blodgett, Phys. Rev., 51, 964 (1937).

49. M. K. Kurnaz and D. K. Schwartz, Langmuir, 12, 4971 (1996).

50. T. Ubukata, S. Morino, T. Seki, and K. Ichimura, Chem. Lett., 1998, 71.

51. T. Ubukata, T. Seki, and K. Ichimura, J. Phys. Chem. B, 104, 4141 (2000).

52. T. Ubukata, T. Seki, S. Morino, and K. Ichimura, J. Phys. Chem. B, 104, 4148 (2000).

53. T. Ubukata, K. Ichimura, and T. Seki, J. Phys. Chem. B, 107, 13831 (2003).
54. K. Kago, T. Seki, R. R. Schucke, E. Mouri, H. Matsuoka, and H. Yamaoka, Langmuir, 18, 3875 (2002).

55. S. Nagano, T. Seki, and K. Ichimura, Chem. Lett., 2000, 612.

56. S. Nagano, T. Seki, and K. Ichimura, Langmuir, 17, 2199 (2001).

57. S. Nagano and T. Seki, J. Am. Chem. Soc., 124, 2074 (2002).

58. J. C. MacDonald and G. M. Whitesides, Chem. Rev., 94, 2383 (1994).

59. T. Kato, Science, 295, 2414 (2002).

60. T. Seki, T. Fukuchi, and K. Ichimura, Bull. Chem. Soc. Jpn., 71, 2807 (1998).

61. T. Seki, T. Fukuchi, and K. Ichimura, Langmuir, 16, 3564 (2000).

62. T. Seki, T. Fukuchi, and K. Ichimura, Langmuir, 18, 5462 (2002).

63. T. Kobayashi, T. Seki, and K. Ichimura, Chem. Commun., 2000, 1193.

64. T. Kobayashi and T. Seki, Langmuir, 19, 9297 (2003).

65. J. Glazer and A. E. Alexander, Trans. Faraday Soc., 47, 401 (1951).

66. T. Kato, H. Akiyama, and M. Yoshida, Chem. Lett., 1992, 565.

67. Y. Urai, C. Ohe, K. Itoh, M. Yoshida, K. Iimura, and T. Kato, Langmuir, 16, 3920 (2000).

68. Q. Huo, S. Russev, T. Hasegawa, J. Nishijo, J. Umemura, G. Puccetti, K. C. Russell, and R. M. Leblanc, J. Am. Chem. Soc., 122, 7890 (2000).

69. T. Kobayashi and T. Seki, Kobunshi Ronbunshu, 60, 569 (2003).

70. C. J. Brown, Acta Cystallogr., 21, 146 (1966).

71. K. Ichimura, Y. Suzuki, T. Seki, A. Hosoki, and K. Aoki, Langmuir, 4, 1214 (1988).

72. M. O’Neill and S. K. Kelly, J. Phys. D: Appl. Phys., 33, R67 (2000).

73. T. Seki, K. Fukuda, and K. Ichimura, Langmuir, 15, 5098 (1999).

74. K. Fukuda, T. Seki, and K. Ichimura, Macromolecules, 35 , 2177 (2002).

75. K. Fukuda, T. Seki, and K. Ichimura, Macromolecules, 35, 1951 (2002).

76. R. D. Miller and J. Michl, Chem. Rev., 89, 1359 (1989).

77. R. H. Tredgold, R. A. Allen, P. Hodge, and E. Khoshdel, J. Phys. D: Appl. Phys., 20, 1385 (1987).

78. A. Natansohn, P. Rochon, M. Pozolét, P. Audet, D. Brown, and S. To, Macromolecules, 27, 2580 (1994).

79. S. R. Andrews, G. Williams, L. Lasker, and J. Stumpe, Macromolecules, 28, 8464 (1995).

80. T. Seki, M. Sakuragi, Y. Kawanishi, T. Tamaki, R. Fukuda, and K. Ichimura, Langmuir, 9, 211 (1993).

81. Th. Geue, A. Ziegler, and J. Stumpe, Macromolecules, 30, 5729 (1997).

82. C. Ruslim and K. Ichimura, Adv. Mater., 13, 37 (2001).

83. K. Ichimura, S. Furumi, S. Morino, M. Kidowaki, M. Nakagawa, M. Ogawa, and Y. Nishiura, Adv. Mater., 12, 950 (2000).

84. K. Ichimura, M. Momose, and T. Fujiwara, Chem. Lett., 2000, 1022. 
85. M. Kidowaki, T. Fujiwara, and K. Ichimura, Chem. Lett., 1999, 643.

86. C. W. Franck, V. Rao, M. M. Despotopoulou, R. F. W. Pease, W. D. Hinsberg, R. D. Miller, and J. F. Rabolt, Science, 273, 912 (1996).

87. C. T. Kresge, M. E. Leonowicz, W. J. Roth, J. C. Varatuli, and J. S. Beck, Nature, 359, 710 (1992).

88. T. Yanagisawa, T. Shimizu, K. Kuroda, and C. Kato, Bull. Chem. Soc. Jpn., 63, 988 (1990).

89. H. Miyata and K. Kuroda, Chem. Mater., 11, 1609 (1999).

90. H. Miyata and K. Kuroda, Adv. Mater., 11, 1448 (1999).

91. Y. Kawashima, M. Nakagawa, T. Seki, and K. Ichimura, Chem. Mater., 14, 2842 (2002).

92. Y. Kawashima, M. Nakagawa, K. Ichimura, and T. Seki, J. Mater. Chem., 14, 328 (2004).

93. A. Hozumi, Y. Yokogawa, T. Kameyama, K. Hiraku, H. Sugimura, O. Takai, and M. Okido, Adv. Mater., 12, 985 (2000).

94. M. Eich, J. H. Wendorff, B. Reck, and H. Ringsdorf, Makromol. Chem., Rapid Commun., 8, 59 (1987).

95. S. Ivanov, I. Yakovlev, S. Kostromin, V. Shibaev, L. Läsker, J. Stumpe, and D. Kreysig, Makromol. Chem., Rapid Commun., 12, 709 (1991).

96. J. Stumpe, L. Müller, and D. Kreysig, Makromol. Chem., Rapid Commun., 12, 81 (1991).

97. A. Natansohn, P. Rochon, J. Gosselin, and S. Xie, Macromolecules, 25, 2268 (1992).

98. T. Ikeda and O. Tsutsumi, Science, 268, 1873 (1995).

99. P. Rochon, E. Batalla, and A. Natansohn, Appl. Phys. Lett., 66, 136 (1995).

100. D. Y. Kim, S. K. Tripathy, L. Li, and J. Kumar, Appl. Phys. Lett., 66, 1166 (1995).

101. P. S. Ramanujam, N. C. R. Holme, and S. Hvilsted, Appl. Phys. Lett., 68, 1329 (1996).

102. J. Kumar, L. Li, X. L. Jiang, D. Y. Kim, T. S. Lee, and S. K. Tripathy, Appl. Phys. Lett., 72, 2096 (1998).

103. C. J. Barrett, P. Rochon, and A. Natansohn, J. Chem. Phys., 109, 1505 (1998).

104. P. S. Ramanujam, M. Pedersen, and S. Hvilsted, Appl. Phys. Lett., 74, 3227 (1999).

105. K. Sumaru, T. Yamanaka, T. Fukuda, and H. Matsuda, Appl. Phys. Lett., 75, 1878 (1999).

106. N. K. Viswanathan, D. Y. Kim, S. Bian, J. Williams, W. Liu, L. Li, L. Samuelson, J. Kumar, and S. K. Tripathy, J. Mater. Chem., 9, 1941 (1999).

107. K. G. Yager and C. J. Barrett, Curr. Opin. Solid State Mater. Sci., 5, 487 (2001).
108. O. N. Oliveira, Jr., J. Kumar, L. Li, and S. K. Tripathy, "Surface-Relief Gratings on Azobenzene-Containing Films," Chap 14 in reference 2, p 429 (2002).

109. T. Fukuda, Kobunshi Ronbunshu, 60, 428 (2003)

110. T. Ubukata, T. Seki, and K. Ichimura, Adv. Mater., 12, 1675 (2000).

111. T. Ubukata, T. Seki, and K. Ichimura, Colloids Surf. A, 113, 198 (2002).

112. T. Ubukata, M. Hara, and T. Seki, Mol. Cryst. Liq. Cryst., 377, 173 (2002).

113. T. Ubukata, T. Seki, and K. Ichimura, Polym. J., 33, 891 (2001).

114. T. Fukuda, H. Matsuda, N. Viswanathan, S. K. Tripathy, J. Kumar, T. Shiraga, M. Kato, and H. Nakanishi, Synth. Met., 102, 1435 (1999).

115. T. Fukuda, H. Matsuda, T. Shiraga, T. Kimura, M. Kato, N. Viswanathan, J. Kumar, and S. K. Tripathy, Macromolecules, 33, 420 (2000).

116. L. Andruzzi, A. Altomare, F. Ciardelli, R. Solaro, S. Hvilsted, and P. S. Ramanujam, Macromolecules, 32, 448 (1999).

117. N. Zettsu, T. Ubukata, T. Seki, and K. Ichimura, Adv. Mater., 13, 1693 (2001).

118. N. Zettsu, T. Fukuda, H. Matsuda, and T. Seki, Appl. Phys. Lett., 83, 4960 (2003).

119. T. Ubukata, M. Hara, K. Ichimura, and T. Seki, $A d v$. Mater., 16, 220 (2004).

120. T. Ubukata, T. Higuchi, N. Zettsu, T. Seki, and M. Hara, Colloid Surf. A, in press.

121. C. B. Murray, D. J. Norris, and M. G. Bawendi, J. Am. Chem. Soc., 115, 8706 (1993).

122. G. S. Hartley, Nature, 140, 281 (1937).

123. G. S. Hartley, J. Chem. Soc., 1938, 633.

124. T. Ikeda and A. Kanazawa, Bull. Chem. Soc. Jpn., 73, 1715 (2000).

125. T. Ikeda, M. Nakano, Y. Yu, O. Tsutsumi, and A. Kanazawa, Adv. Mater., 15, 201 (2003).

126. Y. Yu, M. Nakano, and T. Ikeda, Nature, 425, 145 (2003)

127. M. Yoshikawa, H. Furukawa, K. Horie, and T. Watanabe, Polym. Prepr. Jpn., 52, 777 (2003).

128. Y. Takeoka and M. Watanabe, Adv. Mater., 15, 199 (2003).

129. K. Matsubara, Y. Takeoka, and M. Watanabe, Polym. Prepr. Jpn., 52, 3537 (2003).

130. V. Ajay Mallia and N. Tamaoki, Chem. Soc. Rev., 33, 76 (2004).

131. M. Moriyama, N. Mizoshita, T. Yokota, K. Kishimoto, and T. Kato, Adv. Mater., 15, 1335 (2003).

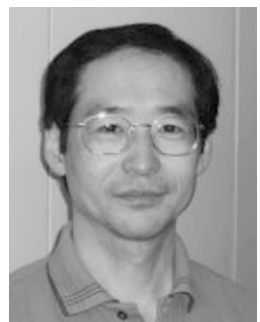

Takahiro Seki was born in Tokyo in 1957. He received Bachelor and Master degrees in Polymer Chemistry from Tokyo Institute of Technology in 1979 and 1982, respectively. He received his Ph.D. from Tokyo Institute of Technology in 1986 on stimuli responsive permeation control in artificial lipid bilayers. He started an academic career at Tokyo Institute of Technology as Research Associate. During 1986-1995 he joined research institutes of Agency of Industrial Science and Technology (MITI) at Tsukuba, Research Institute of Polymers and Textiles (1986-1992) and National Institute of Chemical and Materials Research (1993-1995). Form 1995 to 2002 he was Associated Professor at Chemical Resources Laboratory of Tokyo Institute of Technology, and promoted to Professor at Nagoya University in 2002. He received The SPSJ Wiley Award in 2002. His current research interests include photochromic polymers, organized molecular and polymer films, and liquid crystalline materials. 